\title{
Theory for absorption of ultrashort laser pulses by spheroidal metallic nanoparticles
}

\author{
Nicolas I. Grigorchuk * \\ Bogolyubov Institute for Theoretical Physics, National Academy of Sciences of Ukraine, \\ 14-b Metrologichna Str., Kyiv-143, Ukraine, 03680 \\ Petro M. Tomchuk ${ }^{\dagger}$ \\ Institute for Physics, National Academy of Sciences of Ukraine, \\ 46, Nauky Ave., Kyiv-28, Ukraine, 03680
}

(Dated: June 30, 2018)

\begin{abstract}
The theory for the electric and magnetic fields energy absorption by small metallic particles subjected to the irradiation by ultrashort laser pulses of different duration in the region of surface plasmon excitation is developed. For the particles of the oblate or prolate spheroidal shape there has been found the dependence of the absorbed energy on a number of factors, including a particle radius, a degree of the shape deviation from a spherical one, a pulse duration, the orientation of the magnetic field upon particle, the magnitude of carrier frequency, and the value of a shift of the carrier frequency of a laser ray from the frequency of the surface plasmon excitation in a spherical particle. An appreciable absorption grows at the length of free electron pass large compared to the particle size is established. The phenomenological and kinetic approach is compared each with other.

PACS numbers: 78.67.Bf; 68.49.Jk; 73.63.-b; 75.75.+a
\end{abstract}

\section{INTRODUCTION}

Metallic nanoparticles (MNs) are interesting objects from the viewpoints of physics of condensed state and practical applications. An increase in the local electric fields in the close vicinity of nanoparticles allows the utilization of the MNs as biological markers ${ }^{1}$ and can to lead to entirely new types of biological sensors. 2 The modern optical investigations make it possible to test the optical response of a separate nanoparticle and, thus, to study the properties of separate nanoobjects. $\frac{3}{2}$ This opens the new opportunities for the direct probing of the many-electron dynamics in confined systems. The remarkable results were obtained recently in works. $\underline{4}-\underline{6}$ In recent years, the ultrafast dynamics of electrons in MNs attracts the permanent attention of experimenters. Nanostructures in general have been widely used in modern devices of high-speed electronics and optoelectronics. Owing to the recent achievements in the ultrafast spectroscopy technique, it has become possible to monitor the femtosecond dynamics of the electron gas confined in separate $\mathrm{MNs}^{7}$ The use of short-duration pulses makes it feasible to study the dynamics of fast processes occurring in atoms, molecules, and solids. Pico- and femtosecond resolutions allow to study of vibrational and rotational intramolecular motions, the dynamics of carriers in semiconductor nanostructures, the phase transitions in solids, the processes of formation and breakdown of chemical bonds, etc $\stackrel{8,9}{9}$ The possibility to generate powerful femtosecond pulses has lead to the appearance of

\footnotetext{
*email: ngrigor@bitp.kiev.ua

†email: ptomchuk@iop.kiev.ua
}

a new, rapidly developing branch - the desk-mounted high-energy physics.

When a $\mathrm{MN}$ is placed in the field of a monochromatic electromagnetic (EM) wave and the wavelength is far greater than the particle size, the absorption is observed associated both with the separate and collective kinds of electron motion. The input to the separate absorption gives as an electric component of an EM wave (electric absorption), so the magnetic component (magnetic absorption) as well. The collective electron motion become apparent in so-called surface resonances connected with the excitation of plasmon vibrations (plasmons) of an electron gas. ${ }^{10,11}$ There are three such resonances in an ellipsoidal particle and only one in a spherical particle. In the case of a monochromatic wave, the collective contribution becomes dominant provided that the wave frequency is close to one of the frequencies of plasma resonances, ${ }^{12}$ Numerous applications in decorative painting ${ }^{10}$ and optical devices 13 are based just on the resonances of surface plasmons. However, in spite of the appreciable theoretical efforts, $\stackrel{14}{=}$ a body of problems has remained unsolved hitherto.

In previous works 15 we had shown, that by exposure of metallic nanoparticle to EM wave, the frequency of which is far from plasmon resonances, a separate mechanism becomes actually in absorption. Depending on the shape and size of the particle, as well as on the frequency and polarization of wave, it was shown that, the magnetic absorption can be higher or less than the electric one. The situation is drastically change with using of an ultrashort laser pulses for irradiation, because they contains almost all harmonics. The shorter the pulse, the greater is set of its harmonics. Thus, the ultrashort pulses can excite all the plasma resonances. This is associated with the fact that, in such an ultrashort pulse, 
one can always find the harmonics which correspond to the resonance frequencies of surface plasmons. From the other side, the frequency distribution is very nonuniform (Gaussian) upon the spectra of an ultrashort pulses. How these factors can change the relative input of an electric and magnetic components in absorption, we intend to study below. Another aspect influencing the absorption is associated with the shape of a particle. Earlier, we showed that, in the case of nonspherical MNs, the optical conductivity, which determines both the electric absorption and the half-width of plasma resonances, becomes the tensor quantity. ${ }^{15}$ As far as we know, such a feature in absorption of ultrashort laser pulses by nonspherical MNs was not investigated in details.

In this paper we study for the first time, the peculiar features of the absorption by the MNs of ultrashort pulses, deduced by an electric and magnetic components of EM wave. Such a problems remains less studied, especially for the particles of a nonspherical shape, whose dimensions are less or exceed the electron free path in it, and have attracted a lot of interest of experimenters in recent years. In particular, we will find: (i) the energy absorbed by MNs from ultrashort laser pulses of a different duration; (ii) its dependence on the particle shape, frequency of a carrier wave, pulse duration, and particle sizes; (iii) the shift of the resonance frequency of the absorption with respect to the plasmon frequency in a spherical MN.

The rest of the paper is organized as follows. The model and an initial foundations of problem are presented in Sec. II. Section III contains the study of a plasmon resonances in the MNs. In section IV the study of magnetic absorption is demonstrated using both the phenomenological and the kinetic approaches, and Sec. V contains the conclusions.

\section{MODEL AND STARTING POSITIONS}

Consider a case where an MN is irradiated by a laser pulse, whose electric field is given as

$\mathbf{E}(\mathbf{r}, t)=\mathbf{E}_{0} \exp \left[-\Gamma^{2}\left(t-\frac{\mathbf{k}_{\mathbf{0}} \mathbf{r}}{\omega_{\mathbf{0}}}\right)^{2}\right] \cos \left[\omega_{0}\left(t-\frac{\mathbf{k}_{\mathbf{0}} \mathbf{r}}{\omega_{\mathbf{0}}}\right)\right]$,

where $\Gamma$ is the quantity reciprocal to the pulse duration, $\omega_{0}$ is the carrier frequency of an EM wave, $\left|\mathbf{k}_{\mathbf{0}}\right|=\omega_{0} / c$, and $\mathbf{E}_{0}$ is the maximal value of an electric field in a pulse. In addition to the electric component, the field of a laser pulse contains also the magnetic one which is associated with the former through the corresponding Maxwell equation

$$
\operatorname{rot} \mathbf{E}(\mathbf{r}, t)=-\frac{1}{c} \frac{\partial}{\partial t} \mathbf{H}(\mathbf{r}, t)
$$

We set $\mathbf{E}(\mathbf{r}, t)$ in the form (11) and will find the magnetic component of a pulse, by using Eq. (2). Relation (2) takes the simplest form if one operates with the Fourier components of the above quantities. Actually, for an electric field, e.g., we obtain from Eq. (1)

$$
\begin{aligned}
\mathbf{E}(\mathbf{r}, \omega) & =\int_{-\infty}^{\infty} \mathbf{E}(\mathbf{r}, t) e^{i \omega t} d t=\mathbf{E}_{0} \frac{\sqrt{\pi}}{2 \Gamma} e^{i \mathbf{k}_{\mathbf{0}} \mathbf{r}\left(\frac{\omega}{\omega_{0}}\right)} \\
& \times\left[\exp \left(-\frac{\left(\omega-\omega_{0}\right)^{2}}{4 \Gamma^{2}}\right)+\exp \left(-\frac{\left(\omega+\omega_{0}\right)^{2}}{4 \Gamma^{2}}\right)\right] .
\end{aligned}
$$

Carrying out the Fourier transformation in Eq. (2), one finds connection between Fourier components of the magnetic and electric fields as

$$
\mathbf{H}(\mathbf{r}, \omega)=\frac{c}{i \omega} \operatorname{rot} \mathbf{E}(\mathbf{r}, \omega)
$$

Using the last part of Eq. (3), we find for magnetic field

$$
\begin{aligned}
\mathbf{H}(\mathbf{r}, \omega) & =\frac{\sqrt{\pi}}{2 \Gamma}\left(\mathbf{m} \times \mathbf{E}_{\mathbf{0}}\right) \\
& \times\left(e^{-\frac{\left(\omega-\omega_{0}\right)^{2}}{4 \Gamma^{2}}}+e^{-\frac{\left(\omega+\omega_{0}\right)^{2}}{4 \Gamma^{2}}}\right) e^{i \mathbf{k}_{\mathbf{0}} \mathbf{r}\left(\frac{\omega}{\omega_{0}}\right)}
\end{aligned}
$$

where $\mathbf{m}=\mathbf{k}_{\mathbf{0}} / k_{0}$ is the unit vector directed along the EM wave propagation.

The Fourier component of the electric field amplitude takes the simplest form in the limit $\Gamma \rightarrow 0$ :

$$
\mathbf{E}(\mathbf{r}, \omega)_{\Gamma \rightarrow 0}=\pi \mathbf{E}_{\mathbf{0}} e^{i \mathbf{k}_{0} \mathbf{r}\left(\frac{\omega}{\omega_{0}}\right)}\left[\delta\left(\omega-\omega_{0}\right)+\delta\left(\omega+\omega_{0}\right)\right]
$$

Whereas the electric field of a laser pulse creates the potential electric field $\mathbf{E}_{\text {in }}(\mathbf{r}, t)$ inside an $\mathrm{MN}$, the magnetic field induces the eddy current field $\mathbf{E}_{\text {ed }}(\mathbf{r}, t)$. To calculate the energy absorbed by an MN, we should know the inner fields $\mathbf{E}_{\text {in }}(\mathbf{r}, t)$ and $\mathbf{E}_{\text {ed }}(\mathbf{r}, t)$. To find these quantities, we pay attention to the following feature of the coordinate dependence of the Fourier components (3) and (4): when the characteristic dimension $R$ of a nanoparticle is such that

$$
k_{0} R \ll 1
$$

i.e. the wavelength of the carrier wave is far greater than the MN dimension, the coordinate dependence of the Fourier components $\mathbf{E}(\mathbf{r}, \omega)$ and $\mathbf{H}(\mathbf{r}, \omega)$ can be neglected inside the particle. This means that, in order to determine the inner fields inside an MN, provided that inequality (6) is satisfied, we can set the Fourier components of these fields such as they would be in the spatially uniform fields:

$$
\mathbf{E}(\mathbf{r}, \omega) \rightarrow \mathbf{E}(0, \omega) \text { and } \mathbf{H}(\mathbf{r}, \omega) \rightarrow \mathbf{H}(0, \omega) .
$$

In the case of an nonspherical MN which has, for example, the form of an ellipsoid, this allows us to write the components of the inner electric field as

$$
\left[E_{i n}(\omega)\right]_{j} \approx \frac{E_{j}^{(0)}(0, \omega)}{1+L_{j}[\varepsilon(\omega)-1]}
$$


and the magnetic eddy current field as

$$
\begin{aligned}
{\left[E_{\mathrm{ed}}(\mathbf{r}, \omega)\right]_{x} } & \approx i \frac{\omega}{c} R_{x}^{2} \\
& \times\left[\frac{H_{y}^{(0)}(0, \omega)}{R_{z}^{2}+R_{x}^{2}} z-\frac{H_{z}^{(0)}(0, \omega)}{R_{x}^{2}+R_{y}^{2}} y\right]
\end{aligned}
$$

by analogy with the procedure described in Ref. [16]. The other components of the eddy current field can be obtained from Eq. (9) by means of a cyclic permutation of indices. The notations in formulas (8) and (9) are as follows: $L_{j}(j=x, y, z)$ are the geometrical factors, also known as the depolarization factors, $\stackrel{17}{,} \varepsilon(\omega)=\varepsilon^{\prime}(\omega)+$ $i \varepsilon^{\prime \prime}(\omega)$ is the frequency-dependent complex permittivity of the particle, $R_{x}, R_{y}, R_{z}$ are the ellipsoid semiaxes in the $x, y$, and $z$ directions, respectively.

The linear dependence of the eddy current field on coordinates can be easy seize from Eq. (2), which determined it. If one perform the time Fourier-transform of Eq. (2), than the right-hand side of this equation [as it is seen from both the Eq. (44) and condition [6] ], one can consider as permanent, coordinates-independent quantity. This means that $\operatorname{rot} \mathbf{E}_{e d}(\mathbf{r}, \omega)=$ const. The last equality is satisfy only if

$$
\left[\mathbf{E}_{e d}(\mathbf{r}, \omega)\right]_{j}=\sum_{k=1}^{3} \alpha_{j k}(\omega) x_{k}
$$

e.g., the curly field depends on coordinates linearly. Here $\alpha$ is some matrix does not depended on the $\mathbf{r}$; its components will be further specified.

It is seen from expressions (8) and (9) that the uniform external electric field induces also the uniform electric potential field inside the ellipsoidal MN, whereas the uniform external magnetic field generates the coordinatedependent eddy current field. The inner fields $\mathbf{E}_{\text {in }}(\mathbf{r}, t)$ and $\mathbf{E}_{e d}(\mathbf{r}, t)$ induce the currents inside the MN with the corresponding densities $\mathbf{j}_{\text {in }}(\mathbf{r}, t)$ and $\mathbf{j}_{\text {ed }}(\mathbf{r}, t)$. As a result, the particle absorbs the energy of the EM field of an incident laser wave. The total absorbed energy is sum of electric and magnetic absorption and can be presented $\mathrm{as}^{17}$

$$
\begin{aligned}
w_{t} & =\int_{-\infty}^{\infty} d t W(t)=w_{e}+w_{m}=\int_{-\infty}^{\infty} d t \int_{V} d \mathbf{r} \\
& \times\left[\operatorname{Re} \mathbf{j}_{e}(\mathbf{r}, t) \operatorname{Re} \mathbf{E}_{\mathrm{in}}^{*}(\mathbf{r}, t)\right. \\
& \left.+\operatorname{Re} \mathbf{j}_{\mathrm{ed}}(\mathbf{r}, t) \operatorname{Re} \mathbf{E}_{\mathrm{ed}}^{*}(\mathbf{r}, t)\right],
\end{aligned}
$$

where the integration should be carried out over the whole particle volume. Here, $W(t)$ is the absorbed power. Depending on whether the absorption is brought about by $\mathbf{E}_{\text {in }}(\mathbf{r}, t)$ or $\mathbf{E}_{\text {ed }}(\mathbf{r}, t)$, it is called the electric or magnetic absorption, respectively. In view of the equality $\mathbf{j}^{*}(\omega)=\mathbf{j}(-\omega)$ and

$$
\int_{-\infty}^{\infty} \mathbf{j}_{e}(\mathbf{r}, t) \mathbf{E}_{\mathrm{in}}^{*}(\mathbf{r}, t) d t=\int_{-\infty}^{\infty} \mathbf{j}_{e}(\mathbf{r}, \omega) \mathbf{E}_{\mathrm{in}}^{*}(\mathbf{r}, \omega) \frac{d \omega}{2 \pi}
$$

which follows, in particular, from the Parseval relation for the Fourier integral, ${ }^{18}$ expression (11) can be rewritten as

$$
\begin{aligned}
& w_{t}=\frac{1}{4 \pi} \int_{-\infty}^{\infty} d \omega \int_{V} d \mathbf{r} \\
& \times \quad\left[\mathbf{j}_{e}(\mathbf{r}, \omega) \mathbf{E}_{\mathrm{in}}^{*}(\mathbf{r}, \omega)+\mathbf{j}_{\mathrm{ed}}(\mathbf{r}, \omega) \mathbf{E}_{\mathrm{ed}}^{*}(\mathbf{r}, \omega)+\text { c.c. }\right](1
\end{aligned}
$$

The values of $\mathbf{E}_{\mathrm{in}}(\omega)$ and $\mathbf{E}_{\mathrm{ed}}(\mathbf{r}, \omega)$ can be calculated from formulas (8) and (9), respectively. Thus, the problem remaining to be done is to find the Fourier components of the current densities $\mathbf{j}_{\text {in }}(\mathbf{r}, \omega)$ and $\mathbf{j}_{\text {ed }}(\mathbf{r}, \omega)$. In the general case, the current produced by the inner fields $\mathbf{E}_{\text {in }}(\omega)$ and $\mathbf{E}_{\text {ed }}(\mathbf{r}, \omega)$ at a point $\mathbf{r}$ of the particle can be expressed as the integral over all values of electron velocities $\mathbf{v}$

$$
\mathbf{j}(\mathbf{r}, \omega)=2 e\left(\frac{m}{2 \pi \hbar}\right)^{3} \int_{-\infty}^{\infty} d^{3} v \mathbf{v} f_{1}(\mathbf{r}, \mathbf{v}, \omega) .
$$

Here, $v=|\mathbf{v}|, f_{1}(\mathbf{r}, \mathbf{v}, \omega)$ is the Fourier component of the nonequilibrium distribution function usually considered as an addition to the equilibrium Fermi distribution function $f_{0}(\varepsilon)$ which depends only on the electron kinetic energy $\varepsilon$. Usually, the way to calculate the function $f_{1}(\mathbf{r}, \mathbf{v}, \omega)$ consists in the solution of the corresponding linearized Boltzmann kinetic equation. As a rule, this equation is written for the time-dependent distribution function (see, for example, Ref. 16). Performing the Fourier transformation of this equation with the use of expression (3) and

$$
f_{1}(\mathbf{r}, \mathbf{v}, \omega)=\int_{-\infty}^{\infty} f_{1}(\mathbf{r}, \mathbf{v}, t) e^{i \omega t} d t
$$

we obtain equation for $f_{1}$ :

$$
\begin{gathered}
(\nu-i \omega) f_{1}(\mathbf{r}, \mathbf{v}, \omega)+\mathbf{v} \frac{\partial}{\partial \mathbf{r}} f_{1}(\mathbf{r}, \mathbf{v}, \omega) \\
+e \mathbf{v}\left[\mathbf{E}_{\mathrm{in}}(\omega)+\mathbf{E}_{\mathrm{ed}}(\mathbf{r}, \omega)\right] \frac{\partial}{\partial \varepsilon} f_{0}(\varepsilon)=0,
\end{gathered}
$$

where $\nu$ is the collision frequency in the particle bulk. To move further, we should add the corresponding boundary conditions to Eq. (14). We take assumption of the diffuse reflection of electrons from the inner surface of a particle

$$
\left.f_{1}(\mathbf{r}, \mathbf{v}, \omega)\right|_{S}=0, \quad v_{n}<0
$$

as the boundary conditions. Here, $v_{n}$ is the velocity component normal to the surface $S$. The substantiation of such a boundary conditions and the solution of (14) are presented, in particular, in Ref. 15.

Below we consider separately the peculiarities of the electric absorption when the plasmon resonances are excited by ultrashort laser pulses and the magnetic absorption take place due to magnetic component of the laser wave in more details. 


\section{PLASMON RESONANCES}

To this point, we have considered the general approach which includes both the electric and magnetic absorptions. It was stressed above that the absorption can be either separate or collective. When an MN is in the field of a EM wave and the wave frequency is far from the plasmon resonance, the separate mechanism of absorption prevails. In this case, as was shown in Ref. 15, the electric or magnetic absorption can be dominant depending on a number of factors, namely, on the wave frequency, its polarization, the MN size and shape. In the present section, we concentrate, however, on the features of the absorption of ultrashort laser pulses. In this case, the main contribution to the absorption results from the plasma resonances, i.e. from the collective mechanism, which is a constituent part of an electric absorption. For this reason, we will take into consideration in present section only that part of absorption in Eq. (11), caused by the electric component of the EM field. To find the total energy of electric absorption, we will start from expressions (12) and (13), and determine the form of the function $f_{1}(\mathbf{r}, \mathbf{v}, t)$, by solving Eq. (14) with the corresponding boundary conditions. Then, we will substitute the obtained function into Eq. (13) and calculate the Fourier components of the current density. Finally, by taking into account that the Fourier components of the inner field are described by expression (8), we will substitute the obtained Fourier components of the current density into Eq. (12) with $\mathbf{E}_{e d}=0$. The similar procedure was described in detail in Ref. 15 and, thus, we won't repeat it. We only note that the kinetic equation (14) almost completely coincides with Eq. (27) of work ${ }^{15}$ with the only difference: $\mathbf{E}_{\text {in }}(\omega)$ should be changed by $\mathbf{E}_{\text {loc }}(\omega)$. What is more, the integrand of the integral over frequencies in Eq. (12) formally coincides with the initial expression in Ref. 15. Thus, we can use the results of Ref. 15, in particular formula (75), and write the total energy of an electric absorption in the form

$$
w_{e}=\frac{V}{2 \pi} \sum_{j=1}^{3} \int_{-\infty}^{\infty} \omega^{4} \frac{\sigma_{j j}(\omega)\left|E_{j}^{(0)}(0, \omega)\right|^{2}}{\left(\omega^{2}-\omega_{j}^{2}\right)^{2}+\left(2 \gamma_{j}(\omega)\right)^{2} \omega^{2}} d \omega
$$

where the frequency dependence of $E_{j}^{(0)}(0, \omega)$ is given by expression (3). Here,

$$
\gamma_{j}(\omega)=2 \pi L_{j} \sigma_{j j}(\omega)
$$

is the half-width of a plasmon resonance, which strongly depends on the geometrical shape of a particle and is defined in terms of diagonal components of the optical conductivity tensor $\sigma$. For the different directions $j$, it is governed by the aforementioned factor $L_{j}$. For a spheroidal particle $\left(R_{x}=R_{y} \equiv R_{\perp}\right)$, these components, for example, are reciprocal to the square of the frequency at high frequencies and have the form

$$
\sigma_{\left(\begin{array}{l}
\| \\
\perp
\end{array}\right)}(\omega)=\frac{9}{32 \pi} \frac{v_{\mathrm{F}}}{R_{\perp}}\left(\frac{\omega_{p}}{\omega}\right)^{2}\left(\begin{array}{c}
\eta\left(e_{s}\right) \\
\rho\left(e_{s}\right)
\end{array}\right),
$$

where $v_{\mathrm{F}}$ is the electron velocity on the Fermi sphere, $\omega_{p}$ is the plasma frequency of electron oscillations in a metal, and $\eta\left(e_{s}\right)$ and $\rho\left(e_{s}\right)$ are some smooth functions depending on the spheroid eccentricity $e_{s}$. The analytical dependence of these functions on the ratio between the spheroid semiaxes as welll as, $e_{s}$ can be found in Ref. 15. For a spherical particle, $\eta=\rho=2 / 3$.

The half-widths of plasmon resonances are an important characteristic, since it contains the information about the character of interactions in the system. Through the interaction between surface plasmons and single-particle excitations, the electron scattering gives rise to the decay of surface plasmons, which reveals itself in the broadening of lines of the differential transmission spectra. The surface plasmon decay can occur in different ways, depending on the particle size. Often, the contribution of the Landau decay ${ }^{19}$ to the linewidth, which is dominant in the case of a small particle radius $a$ of the order of $5-20 \AA, 20$ is calculated. At greater particle sizes, the Landau decay competes with the decays caused by other interactions. Recently, we studied ${ }^{21}$ how the relation between the transverse $\gamma_{\perp}$ and longitudinal $\gamma_{\|}$components of the plasmon resonance half-width depends on the degree of ellipsoid's oblateness or prolateness for the frequencies that are higher or lower than the characteristic frequency of electron reflection from the particle walls.

In the case where the plasmon decay is not significant, we can replace the Lorentzian in expression (16) by a $\delta$-function $\operatorname{as}^{22}$

$$
\lim _{\alpha \rightarrow 0} \frac{\alpha}{\alpha^{2}+x^{2}}=\pi \delta(x),
$$

with

$$
x \Rightarrow \omega^{2}-\omega_{j}^{2}, \quad \alpha \Rightarrow 2 \omega \gamma_{j}(\omega)=4 \pi \omega L_{j} \sigma_{j j}(\omega) .
$$

Then, with accounting of Eq.(3), the expression for the absorbed energy takes the form

$$
\begin{aligned}
\left.w_{e}\right|_{\gamma \rightarrow 0} & =\frac{V}{32} \frac{\omega_{p}^{2}}{\Gamma^{2}} \sum_{j=1}^{3} \mid \mathbf{E}_{0 j}\left(\exp \left[-\frac{\left(\omega_{j}-\omega_{0}\right)^{2}}{4 \Gamma^{2}}\right]\right. \\
& \left.+\exp \left[-\frac{\left(\omega_{j}+\omega_{0}\right)^{2}}{4 \Gamma^{2}}\right]\right)\left.\right|^{2},
\end{aligned}
$$

where

$$
\omega_{j}=\sqrt{L_{j}} \omega_{p}
$$

We limit the analysis of Eq. (19) to the case of a spheroidal MN and choose, for convenience, such a polarization of the electric field, which makes it possible to excite the plasmon oscillations of electrons both along and across the spheroid rotation axis. If the field components $\mathbf{E}_{0 \|}$ and $\mathbf{E}_{0 \perp}$ are directed along and across the rotation axis, respectively, then such a polarization is, in the general case,

$$
E_{0 \|} \equiv\left|\mathbf{E}_{0}\right| \cos \theta ; \quad E_{0 \perp}=\left|\mathbf{E}_{0}\right| \sin \theta,
$$




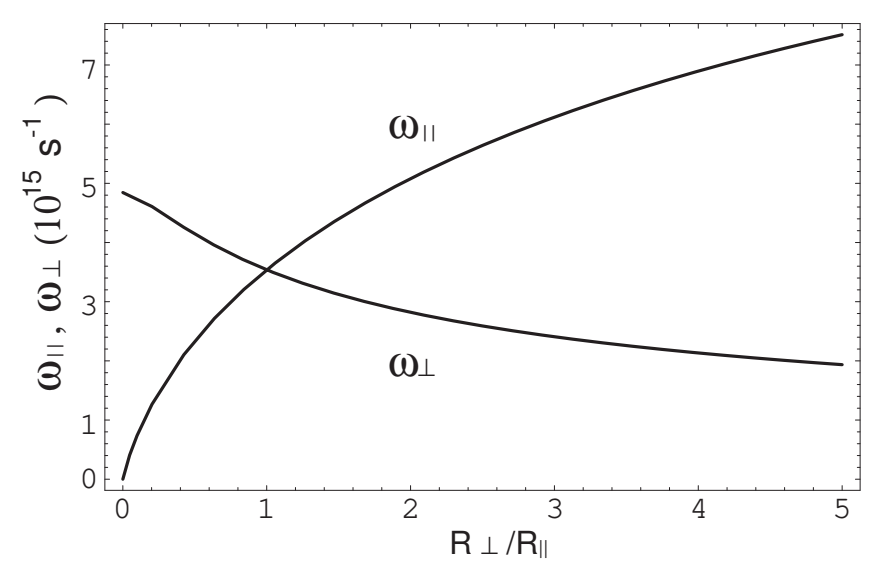

FIG. 1. Dependence of the plasmon resonance frequencies on the form of gold nanoparticle.

where $\theta$ is the angle between the spheroid rotation axis and the vector of the incident electric field strength $\mathbf{E}_{0}$. Then, frequencies (20) can be rewritten as the frequencies of electron oscillations along and across the axis of rotation:

$$
\omega_{\|, \perp}=\sqrt{L_{\|, \perp}} \omega_{p}
$$

which, for the given $R_{\|, \perp}$ will be the frequencies of the corresponding plasmon resonances. The dependence of the plasmon resonance frequencies on the particle form is illustrated in Fig. 1 for Au.

We will use the electromagnetic radiation energy $w$ traversing the nanoparticle during the total duration of the pulse as a scale. This energy at normal light incidence, we define as

$$
w=s c \int_{-\infty}^{\infty}\left|\mathbf{E}_{\mathbf{0}}(0, t)\right|^{2} d t=\frac{s c}{2 \Gamma} \sqrt{\frac{\pi}{2}}\left(1+e^{-\frac{\omega_{0}^{2}}{2 \Gamma^{2}}}\right)\left|\mathbf{E}_{\mathbf{0}}\right|^{2},
$$

where $c$ is the light velocity, and

$$
s=\pi\left(R_{\perp}^{2} R_{\|}\right)^{2 / 3}
$$

is the particle section. In what follows, for the sake of illustration, we will calculate the quantity

$$
S_{e}=\frac{w_{e}}{2 w}
$$

which is the ratio of the energy absorbed by a unit of the MN volume to the energy traversed the nanoparticle. The ratio between particle volume and particle section is

$$
\frac{V}{s}=\frac{4}{3} R
$$

where

$$
R=\sqrt[3]{R_{\perp}^{2} R_{\|}}
$$

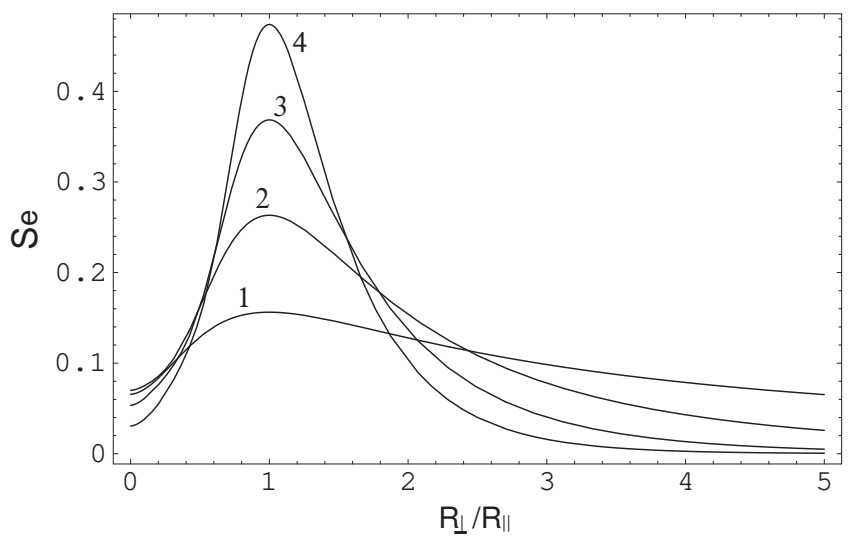

FIG. 2. Dependence of the energy absorbed by a spheroidal gold particle with $R=\left(R_{\perp}^{2} R_{\|}\right)^{1 / 3}=200 \AA$ at the plasmon resonance frequency on the ratio between the spheroid semiaxes for different values of $\Gamma, \mathrm{s}^{-1}: 2.637 \times 10^{15}(1), 1.582 \times 10^{15}(2), 1.13 \times 10^{15}$ (3), and $0.879 \times 10^{15}(4)$. $\theta=\pi / 4$.

Figure 2 shows the dependence of $S_{e}$ on the degree of ellipsoid's oblateness or prolateness in the case where $\theta=$ $\pi / 4$ and the frequency equals that of surface plasmons of a spherical particle $\omega_{0}=\omega_{p} / \sqrt{3} \equiv \Omega$. Here and below, the calculations are carried out with the use of formulas (19) and (21) - (27), given electron density in the $\mathrm{Au}$ particle ${ }^{23} n \approx 5.9 \times 10^{22} \mathrm{~cm}^{-3}, \omega_{p}=\left(4 \pi n e^{2} / m\right)^{1 / 2} \approx$ $1.37 \times 10^{16} \mathrm{~s}^{-1}$ and $\Omega \approx 7.91 \times 10^{15} \mathrm{~s}^{-1}$. The numerical data for $\Gamma$ are taken here and below (in illustrations) from the Table 1. It is convenient to measure $\Gamma$ in the fractions of the carrier frequency $\omega_{0}$. Then, the duration of a laser pulse can be set in the carrier wave periods or wavelengths, that is $1 / \Gamma_{n}=n / \omega_{0} \sim n \lambda_{0}$, where $n$ is an arbitrary integer. Thus, for the region of plasmon resonances (when $\omega_{0} \equiv \Omega$ ), each wavelength, which is a multiple of $\lambda_{0}$, can be brought into correspondence with a certain pulse duration.

TABLE I. Correspondence between the value inverse to the pulse duration and the wavelength of a carrier wave in the region of plasmon resonances for $\mathrm{Au}$ particle in vacuum.

\begin{tabular}{cc}
\hline \hline$\Gamma \times 10^{15}, \mathrm{~s}^{-1}$ & $\lambda$ \\
\hline 7.911 & $\lambda_{0}$ \\
2.637 & $3 \lambda_{0}$ \\
1.582 & $5 \lambda_{0}$ \\
1.130 & $7 \lambda_{0}$ \\
0.879 & $9 \lambda_{0}$ \\
0.791 & $10 \lambda_{0}$ \\
0.527 & $15 \lambda_{0}$ \\
0.396 & $20 \lambda_{0}$ \\
\hline \hline
\end{tabular}

As follows from curves 1-4, the laser pulses of longer duration are absorbed more effectively. As for the shape dependence of the absorption, the maximum of absorption is achieved at $R_{\perp} / R_{\|}=1$, i.e., when the particle is in vacuum and have the spherical shape. If the particle is embedded in the dielectric matrix with $\varepsilon>1$, then maximum is shifted to the red side of the spectra range, pro- 
portionally to the value of $\varepsilon$. For example, for Au particle embedded in the glass matrix with $\varepsilon=7$, the plasmon resonance pick occurs at the frequency $\Omega_{m} \simeq \Omega-0.55 \Omega$.

The calculation of $S_{e}$ was also carried out by means of the numerical integration in formula (16) for the various particle radii $R$ within the range of $R=\left(R_{\|} R_{\perp}^{2}\right)^{1 / 3}=$ $50 \div 400 \AA$. On the whole, it turned out that the value of $S_{e}$, calculated numerically, is (i) slightly smaller than the values obtained on the basis of formula (19) and (ii) depends on $R$ very weakly for various ${ }^{24} \Gamma$. At the same time, the latter dependence is more pronounced at smaller $\Gamma$ provided that $R$ is not great $(R=50 \div 150$ $\AA)$. For example, for particle with $R=100 \AA$ at $\Gamma=0.879 \times 10^{15} \mathrm{~s}^{-1}$, the highest possible values of $S_{e}$ calculated analytically with the use of Eq. (19) exceed those numerically determined from formula (16) by about $7 \%$. For greater $R$ and $\Gamma$, this percentage decreases. For this reason, we will rest in the further calculations of $S_{e}$ on the evaluations performed according to formula (19).

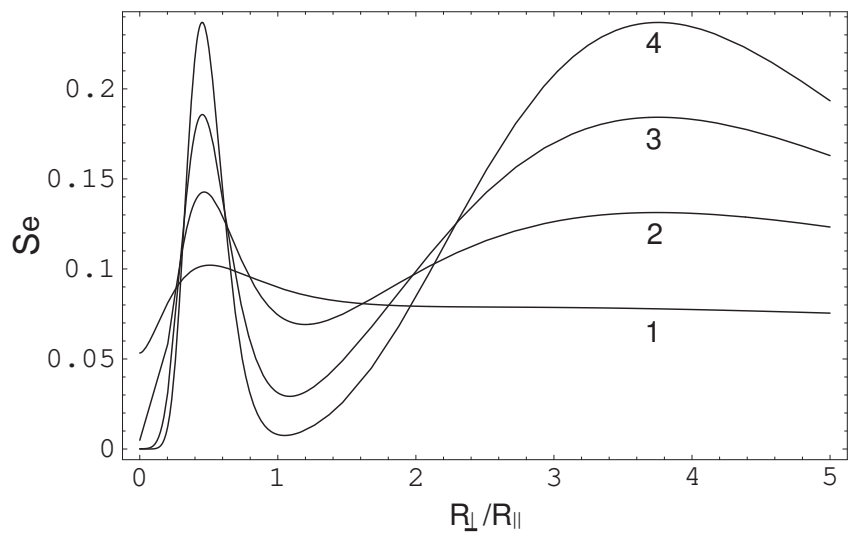

FIG. 3. The same as in Fig. 1, for the frequency $\omega_{0}=5.4 \times$ $10^{15} \mathrm{~s}^{-1}$.

As soon as the carrier frequency deviates from that of the surface plasmon in a spherical particle, the peak of absorbed energy depending on the ratio $R_{\perp} / R_{\|}$splits into the two ones: the first maximum corresponds to the prolate particles $\left(R_{\perp} / R_{\|}<1\right)$, whereas the second - to the oblate particles $\left(R_{\perp} / R_{\|}>1\right)$. This fact is illustrated in Fig. 3 for the frequency $\omega_{0}=5.4 \times 10^{15} \mathrm{~s}^{-1}$ and various values of the incident pulse duration.

If the pulse duration is fixed, then, changing the frequency of a carrier wave, we can trace the evolution of the dependence of the maximum of absorbed energy on the MN shape. Figures $4(a)$ and 4(b) illustrate such a dependencies. For comparison, the absorption curve at a frequency $\omega_{0}=\Omega$, where $\Omega$ is the resonance frequency of a spherical MN, is shown (curve 1 in each part of the figure). For the frequencies exceeding $\Omega$ (see Fig. 4(a)), the absorption maximum first shifts towards the side of more oblate particles with increase in $\omega_{0}$ and then splits into the two peaks: the first one is observed at $R_{\perp} / R_{\|}>1$, whereas the second one - at $R_{\perp} / R_{\|}<1$. Figure $4(b)$ demonstrates the data calcu- lated for the frequencies lower than $\Omega$, with the calculations carried out for the same values of the pulse duration. As the frequency gets lower, the absorption maximum shifts towards the side of more prolate particles and, afterwards, splits into two, as in the former case. As $\omega_{0}$ deviates from the resonance frequency, the peak of the absorbed energy first decreases in absolute value, then splits and finally, as the carrier frequency strongly differs from $\Omega$, the values of both emerged peaks reach the plateau.

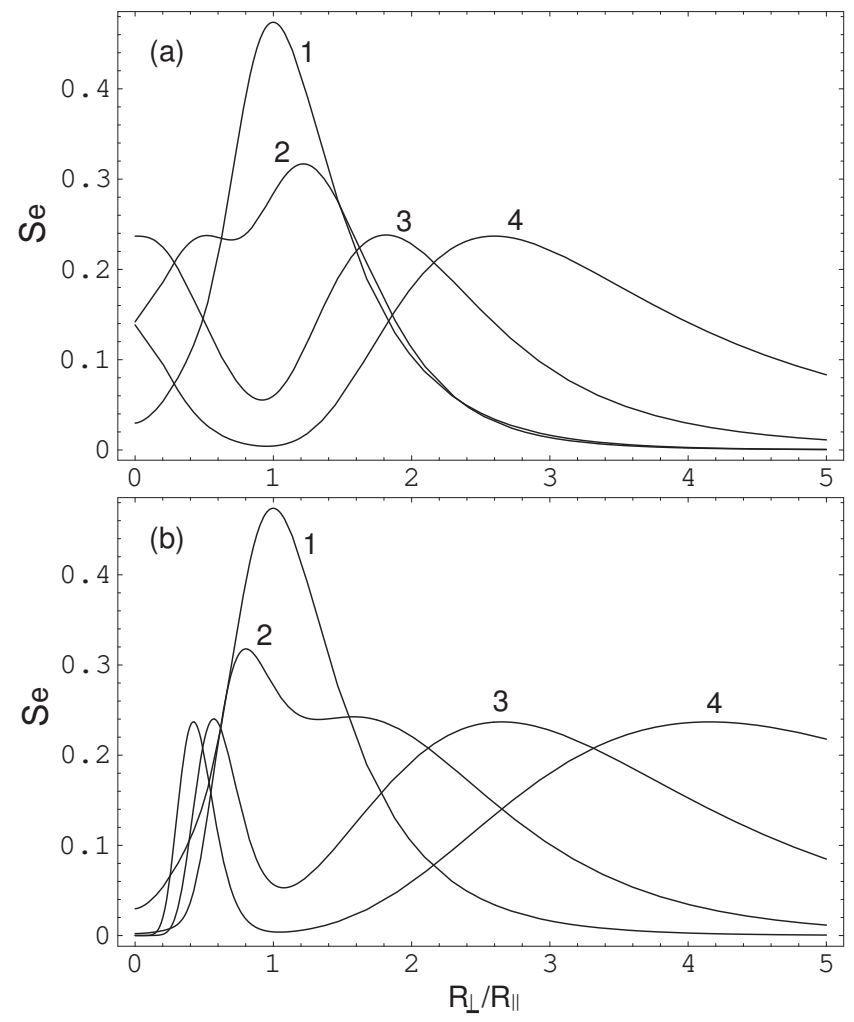

FIG. 4. The energy absorbed by a spheroidal gold particle with $R=$ $\left(R_{\perp}^{2} R_{\|}\right)^{1 / 3}=200 \AA$ as the function of the degree of its oblateness or prolateness for $\Gamma=0.879 \times 10^{15} \mathrm{~s}^{-1}$, at the frequencies $\omega_{0}$ higher than that of the plasmon resonance $\Omega$ of a spherical MN (a): $\omega_{0}=8.8 \times 10^{15} \mathrm{~s}^{-1}(2), 9.7 \times 10^{15} \mathrm{~s}^{-1}(3)$, and $10.6 \times 10^{15}$ $\mathrm{s}^{-1}(4)$ and lower than $\Omega(b): \omega_{0}=7.0 \times 10^{15} \mathrm{~s}^{-1}(2), 6.1 \times 10^{15}$ $\mathrm{s}^{-1}(3)$, and $5.2 \times 10^{15} \mathrm{~s}^{-1}(4) . \theta=\pi / 4$. Curves 1 in both parts of the figure correspond to the case $\omega_{0}=\Omega$.

These results are in compliance with the correlation, established by us earlier, $\stackrel{21}{\hookrightarrow}$ between the geometrical shape of a nanoparticle and the frequency of the light absorption, depending on them, the rapid growth in the strength of light pressure on a particle is observed.

Let us choose the prolateness or oblateness of a nanoparticle fixed and trace how the absorption changes as a function of the carrier frequency. In the case of a prolate $\mathrm{MN}$, with increase in the duration of the incident laser pulse, one observes not only the growth of the peak of absorbed energy, but also its splitting (as $\Gamma$ becomes higher than a certain critical value) into two peaks (a doublet) located on the opposite sides of the line 
$\omega_{0} / \Omega=1$ (Fig. 5), where $\Omega$ corresponds to the resonance frequency of a spherical particle. It is worth to note that the peaks at $\omega_{0}<\Omega$ and $\omega_{0}>\Omega$ correspond to the plasmon resonance frequencies $\omega_{\|}$and $\omega_{\perp}$, respectively (see Eq. (22) and Fig. 1), whereas the inverse situation is characteristic of oblate particles: the former peak corresponds to $\omega_{\perp}$ and the latter - to $\omega_{\|}$(see Fig. 1).

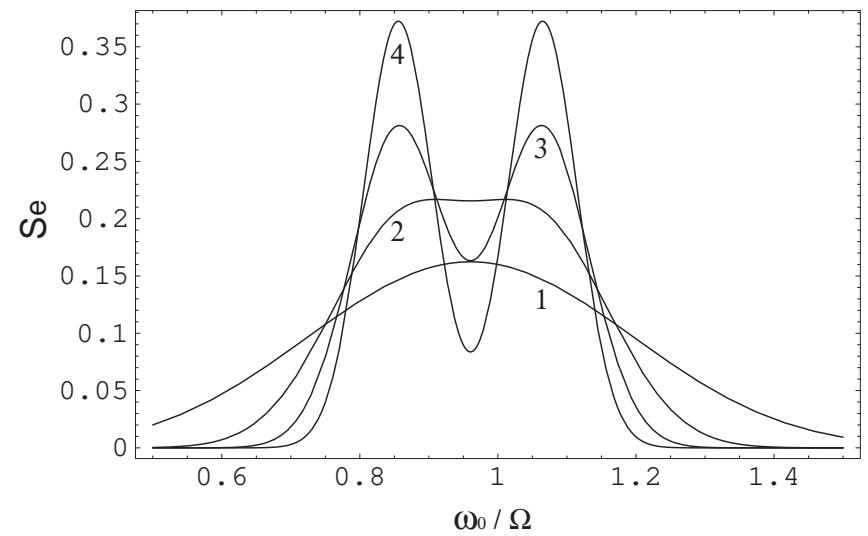

FIG. 5. Dependence of the energy absorbed by a prolate $\left(R_{\perp} / R_{\|}=\right.$ 0.7 ) gold particle with $R=\left(R_{\perp}^{2} R_{\|}\right)^{1 / 3}=200 \AA$ on the frequency of a carrier wave for different values of $\Gamma, \mathrm{s}^{-1}: 1.582 \times 10^{15}(1)$, $0.791 \times 10^{15}(2), 0.527 \times 10^{15}(3)$, and $0.396 \times 10^{15}(4)$ (taken from Table 1). $\theta=\pi / 4$.

Thus, contrary to the case of spherical particles, the spheroidal MNs, either prolate or oblate, exhibit a doublet in the spectrum of absorption provided that $\Gamma$ is sufficiently small. The doublet originates from two plasmon resonances excited in particles by ultrashort laser pulses. At $\theta=\pi / 4$, both the peaks of the doublet have the same height, which, being practically independent of a degree of the particle prolateness or oblateness, strongly depends on the pulse duration. In the case of prolate MNs, a minimum of the dip between the absorption peaks is achieved at frequencies $\omega_{0}$ lower than $\Omega$ and shifts towards the longer waves with increase in the prolateness degree. The other tendency is characteristic of oblate MNs: the minimum is observed at frequencies higher than $\Omega$ and, as the oblateness degree increases, it first shifts towards the shorter waves and then, at sufficiently high oblateness degrees, the movement changes its direction.

The peaks of absorption display the similar behavior. As the degree of the MN prolateness increases, the distance between the peaks of the doublet grows due to the more drastic shift of the peak, caused by the plasmon resonance at the frequency $\omega_{\|}$, toward the longer waves. At the same time, the height of the peaks remains constant provided that the degree of prolateness is small enough. Quite similar behavior is also characteristic of oblate MNs: the distance between the peaks of the doublet grows with increase in the degree of the MN oblateness. The reason for this is either the more drastic shift of the peak, caused by the plasmon resonance at $\omega_{\|}$, toward the highfrequency side of the spectrum (at sufficiently small degrees of oblateness) or the reverse movement of the peak, caused by the plasmon resonance at $\omega_{\perp}$ (at higher degrees of oblateness). The height of the peaks remains constant as the degree of oblateness keeps small enough.

Under various experimental conditions, a small shift, either blue or red, with respect to the value $\omega_{0}=\Omega$ has been observed. A number of microscopic approaches has been developed to explain such a shift (see, for example, Ref. 11). The most successful approaches are thought to be those built on the basis of a jelly model with the use of the theory of linear response in the limits of time-dependent local density $\underline{25}$ However, these approaches don't account for the shift caused by a change in the particle shape. It is the most important feature of our approach that results in the facts that the shape of the MNs specifies the frequency, at which the resonance absorption occurs, and the shift of the plasmon absorption peak is associated with a change in the particle shape.

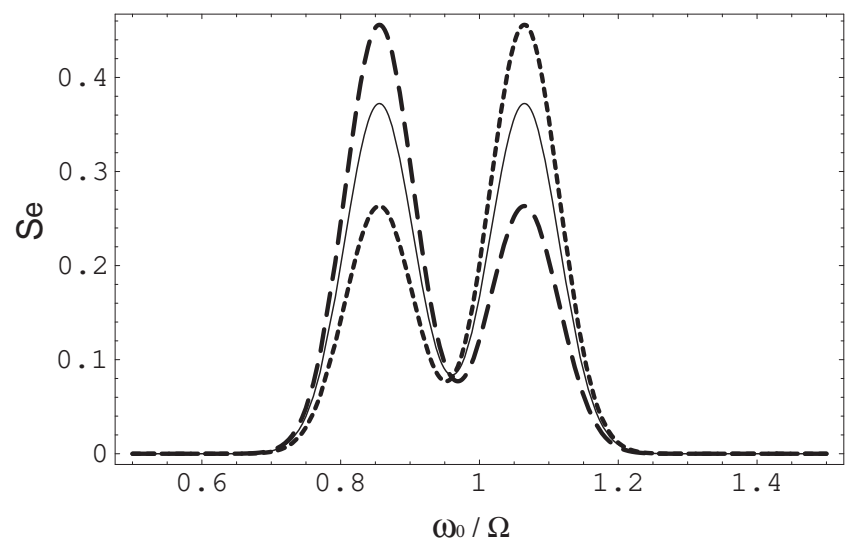

FIG. 6. The energy absorbed by a prolate $\left(R_{\perp} / R_{\|}=0.7\right)$ gold particle with $R=\left(R_{\perp}^{2} R_{\|}\right)^{1 / 3}=200 \AA$ as the function of the frequency of a carrier wave for $\Gamma=0.396 \times 10^{15}, \mathrm{~s}^{-1}$ and different angles of the laser pulse incidence: $\theta=\pi / 4-$ solid line, $\theta=\pi / 3-$ dotted line, and $\theta=\pi / 6$ - dashed line.

The relative height of the peaks in the doublet can be tuned by means of changing the angle of the laser pulse incidence. Figure 6 allows one to trace how the plasmon absorption along and across the spheroid rotation axis changes as the angle of incidence $\theta$ is reduced from $\pi / 3$ to $\pi / 6$. For convenience, we consider the prolate particle with $R_{\perp} / R_{\|}=0.7$, as in Fig. 5. As is seen in Fig. 6, the peak intensities are the same at $\theta=\pi / 4$, but they become different as $\theta$ deviates from this value. So, for example, at $\theta=\pi / 3$, the peak associated with the resonance at $\omega_{\|}$is characterized by a reduced intensity, contrary to the peak associated with $\omega_{\perp}$, which is characterized by an increased intensity. At $\theta=\pi / 6$, however, the inverse situation is observed. It is obviously that, whereas the intensity of the peak at $\omega_{\|}$reaches a maximum at $\theta=0$ and turns into the zero at $\theta=\pi / 2$, the peak at $\omega_{\perp}$ has maximum intensity at $\theta=\pi / 2$ and minimum one - at $\theta=0$.

Lastly, we consider the qualitatively different limit 
transition when the width of an incident laser pulse is sufficiently large. To do this, we use the next representation for $\delta$-function 22

$$
\delta(x)=\frac{1}{\sqrt{\pi}} \lim _{\alpha \rightarrow 0} \frac{1}{\alpha} \exp \left(-x^{2} / \alpha^{2}\right),
$$

and calculate, according to Eq. (3), the value of $\left|E_{j}^{(0)}(0, \omega)\right|^{2}$, which is included in Eq. (16)

$$
\left|E_{j}^{(0)}(0, \omega)\right|^{2} \simeq 2 E_{0 j}^{2}\left(\frac{\pi}{2}\right)^{3 / 2} \frac{1}{\Gamma}\left[\delta\left(\omega-\omega_{0}\right)+\delta\left(\omega+\omega_{0}\right)\right] .
$$

Now relations (16) and (28) yield

$$
\left.w_{e}\right|_{\Gamma \rightarrow 0}=\frac{2 V}{\pi}\left(\frac{\pi}{2}\right)^{3 / 2} \frac{1}{\Gamma} \sum_{j} \frac{\omega_{0}^{4} \sigma_{j j}\left(\omega_{0}\right) E_{0 j}^{2}}{\left(\omega_{0}^{2}-\omega_{j}^{2}\right)^{2}+\left(2 \gamma_{j}\left(\omega_{0}\right)\right)^{2} \omega_{0}^{2}} .
$$

As is seen from Eq. (29), the absorbed energy comparing with Eq. (19) is proportional only to the first order of the pulse duration $1 / \Gamma$. Expression (29) divided by the pulse duration gives us the value of the energy which is absorbed by a nanoparticle on average in a unit of time at its irradiation by long-duration pulses. This quantity differs by the factor $2 \sqrt{\pi / 2}$ from the analogous quantity obtained for the absorption of a spherical wave. 26
The energy absorbed by spheroidal MP in the case of broad pulses is given from Eqs. (29), (18) by

$$
\left.w_{e}\right|_{\Gamma \rightarrow 0}=\frac{9}{32} \frac{\left|\mathbf{E}_{0}\right|^{2}}{\sqrt{2 \pi}} \frac{V}{\Gamma} \omega_{0}^{2} \omega_{p}^{2} \frac{v_{F}}{R_{\perp}}\left(\frac{\eta\left(e_{s}\right) \cos ^{2} \theta}{\left(\omega_{0}^{2}-\omega_{\|}^{2}\right)^{2}+\left[2 \gamma_{\|}\left(\omega_{0}\right)\right]^{2} \omega_{0}^{2}}+\frac{\rho\left(e_{s}\right) \sin ^{2} \theta}{\left(\omega_{0}^{2}-\omega_{\perp}^{2}\right)^{2}+\left[2 \gamma_{\perp}\left(\omega_{0}\right)\right]^{2} \omega_{0}^{2}}\right)
$$

where

$$
\gamma_{\left(\begin{array}{l}
\| \\
\perp
\end{array}\right)}\left(\omega_{0}\right)=2 \pi L_{\left(\begin{array}{l}
\| \\
\perp
\end{array}\right)} \sigma_{\left(\begin{array}{l}
\| \\
\perp
\end{array}\right)}\left(\omega_{0}\right)
$$

and $L_{\|}, L_{\perp}$ are the geometrical factors along and across the long spheroid axis of a revolution, correspondingly.

\section{MAGNETIC ABSORPTION}

If the frequency of a monochromatic EM wave is far from a plasmon resonance, then a separate mechanism plays the main role in the MN absorption. As we have already pointed out in this case, either an electric or magnetic absorption can dominate. But if the size of a spherical particle, for instance, is larger than $50 \AA$, then the magnetic absorption begin to exceed the electric one even at frequencies less than the frequency of electron reflections from opposite walls of a particle $\frac{15}{1 n}$ this section, we will consider in detail, the behavior of the magnetic absorption at frequencies close to the plasmon resonances of the MN.

In accordance with Eqs. (11) and (12), the magnetic field energy can be presented in an integral form as follows

$$
w_{m}=\frac{1}{2} \int_{-\infty}^{\infty} \frac{d \omega}{2 \pi} \int_{V} d \mathbf{r}\left[\mathbf{j}_{e d}(\mathbf{r}, \omega) \mathbf{E}_{e d}^{*}(\mathbf{r}, \omega)+c . c .\right] .
$$

The value of $\mathbf{E}_{e d}(\mathbf{r}, \omega)$ is already known for us from Eq. (9). In order to calculate $\mathbf{j}_{e d}(\mathbf{r}, \omega)$ with the use of the formula (13), it is necessary first to find the distribution function for electrons. For this purpose, we would seek for solutions of Eq. (14), where $E_{\text {in }}(\omega)=0$. To satisfy both the equation (14) and the boundary conditions (15), we should pass to the deformed variables

$$
x_{i}^{\prime}=x_{i} \frac{R}{R_{i}}, \quad v_{j}^{\prime}=v_{j} \frac{R}{R_{j}} .
$$

Then an ellipsoid can be represented as a sphere of the same volume with the radius $R$, and the solution of Eq. (14) we can write in the form

$$
\begin{aligned}
f_{1}\left(\mathbf{r}^{\prime}, \mathbf{v}^{\prime}, \omega\right) & =-\frac{e}{R^{2}} \frac{\partial f_{0}}{\partial \varepsilon} \sum_{i, j=1}^{3} \alpha_{i j}(\omega) v_{j}^{\prime} R_{j} R_{i} \\
& \times\left(x_{i}^{\prime}+v_{i}^{\prime} \frac{\partial}{\partial(\nu-i \omega)}\right) \frac{1-e^{-(\nu-i \omega) t^{\prime}\left(\mathbf{r}^{\prime}, \mathbf{v}^{\prime}\right)}}{\nu-i \omega},
\end{aligned}
$$

where

$$
t^{\prime}\left(\mathbf{r}^{\prime}, \mathbf{v}^{\prime}\right)=\frac{1}{v^{\prime 2}}\left[\mathbf{r}^{\prime} \mathbf{v}^{\prime}+\sqrt{\left(R^{2}-r^{\prime 2}\right) v^{\prime 2}+\left(\mathbf{r}^{\prime} \mathbf{v}^{\prime}\right)^{2}}\right] .
$$

In Eq. (33), the energy derivative of the distribution function in zero approximation in a small ratio of $k_{B} T / \varepsilon_{F}$ can be replaced by

$$
\frac{\partial f_{0}}{\partial \varepsilon} \rightarrow-\delta\left(\varepsilon-\varepsilon_{F}\right),
$$


where $\varepsilon_{F}$ it the Fermi energy. The diagonal components of the matrix $\alpha_{j j}$ are $\alpha_{j j}=0$. The nondiagonal ones can be expressed in terms of appropriate components of the magnetic field. For example,

$$
\alpha_{x y}(\omega)=-i \frac{\omega}{c} \frac{R_{x}^{2}}{R_{x}^{2}+R_{y}^{2}} H_{z}^{(0)}(0, \omega) .
$$

The other two components of the matrix $\alpha$ can be obtained from Eq. (36) by means of a cyclic index permutation. The remaining three components one can find using the antisymmetric character of the $\alpha$, namely, taking into account the property $\alpha_{x y}(\omega)=-\alpha_{y x}(\omega)$.

Thus, to find the energy of the magnetic absorption it is necessary to undertake step-by-step the following procedure: to inset the obtained function $f_{1}(\mathbf{r}, \mathbf{v}, t)$ into Eq. (13) and then, taking into account the expression (9), to put the Fourier components for both the current density and the curly field into Eq. (32).

One can escape the above procedure for energy calculation, using the phenomenological approach for an estimation of current. It means that the absorption of a particle can be written down in a general form with real and imaginary parts of a dielectric permeability, and thus it is not necessity to solve the kinetic equation (14) with the boundary conditions (15).

\section{A. Phenomenological approach}

Assume that the particle size is much more greater than the electron free pass within it. Then, the curly current can be expressed in terms of a curly field by means of

$$
\mathbf{j}_{\mathrm{ed}}(\omega)=\sigma_{\mathrm{m}}(\omega) \mathbf{E}_{\mathrm{ed}}(\omega),
$$

and the expression (32) can be rewritten in the form

$w_{m}=\frac{1}{2} \sum_{j=1}^{3} \int_{-\infty}^{\infty}\left[\sigma_{m}(\omega)+\sigma_{m}^{*}(\omega)\right] \frac{d \omega}{2 \pi} \int_{V}\left|\mathbf{E}_{e d}^{j}(\mathbf{r}, \omega)\right|^{2} d \mathbf{r}$.

One can change here the integration over an ellipsoid volume $V$ by the integration over the sphere of an equivalent volume. Then, it is easy to show, that

$$
\begin{aligned}
& \int_{V} x^{2} d \mathbf{r}=V \frac{R_{x}^{2}}{5}, \\
& \int_{V} y^{2} d \mathbf{r}=V \frac{R_{y}^{2}}{5}, \quad \int_{V} z^{2} d \mathbf{r}=V \frac{R_{z}^{2}}{5}, \\
& \int_{V} x y d \mathbf{r}=\int_{V} y z d \mathbf{r}=\int_{V} z x d \mathbf{r}=0 .
\end{aligned}
$$

Further, we will restrict ourselves with consideration of the metal nanoparticles having the spheroid shape $\left(R_{x}=\right.$
$\left.R_{y} \equiv R_{\perp}, R_{z} \equiv R_{||}\right)$. Using Eqs. (9) and (38), the sum of integrals over a nanoparticle volume will be

$$
\begin{aligned}
& \sum_{j=1}^{3} \int_{V}\left|\mathbf{E}_{e d}^{j}(\mathbf{r}, \omega)\right|^{2} d \mathbf{r}=\frac{V}{5}\left(\frac{\omega}{c}\right)^{2} \\
\times & \left(\frac{R_{\perp}^{2}}{2} \mathbf{H}_{\| \mid}^{2}(0, \omega)+\frac{R_{||}^{2} R_{\perp}^{2}}{R_{||}^{2}+R_{\perp}^{2}} \mathbf{H}_{\perp}^{2}(0, \omega)\right) .
\end{aligned}
$$

Here, $\mathbf{H}_{\|}(0, \omega)$ is the intensity of the magnetic field along the spheroid axis of a revolution, and $\mathbf{H}_{\perp}(0, \omega)-$ transverse to it. It is worth to pay attention that the magnetic absorption depends on the magnetic field polarization in the same way as the electric absorption depends on the electric field polarization $\cdot \frac{17}{}$ Moreover, one can generalize the expression (39) to the case of an arbitrary coordinate systems if the field components are represented as

$$
\mathbf{H}_{\perp}^{2}=\mathbf{H}^{2}-\mathbf{H}_{||}^{2}, \quad \mathbf{H}_{||}=(\mathbf{H} \cdot \mathbf{n}) \mathbf{n},
$$

where $\mathbf{n}$ is a unit vector directed along the spheroid axis of a revolution. Then with an account of Eq. (39), the expression (37) transforms into

$$
\begin{aligned}
w_{m} & =\frac{V}{10} \frac{R_{||}^{2} R_{\perp}^{2}}{R_{||}^{2}+R_{\perp}^{2}} \int_{-\infty}^{\infty}\left[\sigma_{m}(\omega)+\sigma_{m}^{*}(\omega)\right]\left(\frac{\omega}{c}\right)^{2} \\
& \times\left(\mathbf{H}^{2}(0, \omega)+\frac{1}{2}\left(1-\frac{R_{\perp}^{2}}{R_{||}^{2}}\right)(\mathbf{H}(0, \omega) \mathbf{n})^{2}\right) \frac{d \omega}{2 \pi} .
\end{aligned}
$$

The field $\mathbf{H}(0, \omega)$ in Eq. (41) is defined by the expression (4), and $\sigma_{m}$ for a metallic nanoparticle in the phenomenological case is

$$
\sigma_{m}(\omega)=\frac{1}{4 \pi} \frac{\omega_{p}^{2}}{\nu-i \omega}
$$

where $\omega_{p}=4 \pi n e^{2} / m$ is the plasma frequency of the electron vibrations in a metal.

Next, we will perform calculations for certain polarizations of the magnetic component of the EM wave with respect to the particle orientation. We will consider the following two cases:

(i) Let us direct the vector of magnetic field transverse to the spheroid axis. Then, the second item under the integral in Eq. (41) vanishes, and after substituting Eqs. (41) and (42) into Eq. (41), we get for the absorbed energy the expression

$$
\begin{aligned}
w_{m \perp} & =\frac{V}{80 \Gamma^{2}} \frac{\nu \omega_{p}^{2}}{c^{2}} \frac{R_{||}^{2} R_{\perp}^{2}}{R_{||}^{2}+R_{\perp}^{2}}\left|\mathbf{E}_{0}\right|^{2} \\
& \times \int_{-\infty}^{\infty} \frac{\omega^{2}}{\nu^{2}+\omega^{2}} f(\omega) \frac{d \omega}{2 \pi}
\end{aligned}
$$


where

$$
f(\omega)=\left(\exp \left[-\frac{\left(\omega-\omega_{0}\right)^{2}}{4 \Gamma^{2}}\right]+\exp \left[-\frac{\left(\omega+\omega_{0}\right)^{2}}{4 \Gamma^{2}}\right]\right)^{2} .
$$

Our attempt to fulfil the integration in Eq. (43) in an analytical form exactly was not successful, but using the fact that the frequency of electron collisions with phonons $\nu$ is a small quantity comparing to the frequencies $\omega \approx$ $\omega_{0}$, which yield the main contribution to the integral, one can neglect by $\nu$ under the integral in Eq. (431). For instance, $\omega_{0} \sim 10^{15} \mathrm{~s}^{-1}$ and for $\mathrm{Au} \nu_{0^{\circ} \mathrm{C}} \simeq 3.39 \times 10^{13} \mathrm{~s}^{-1}$, that comes from the formula for electroconductivity of metal $\sigma=1 / \rho \simeq n e^{2} /(m \nu)$, with $\rho_{0^{\circ} \mathrm{C}}=2.04 \times 10^{-6} \Omega \mathrm{cm}$ (Ref. 27). Then, one gets the result equal to twice the integral of

$$
I_{\nu}=\int_{\nu}^{\infty} f(\omega) d \omega .
$$

This integral can be calculated analytically even in the pointed limits. However, it is easy to show, that the result of the integration practically is not changed if one brings the lower integration limit in Eq. (45) down to the zero. This means only that the integral between limits $[0, \nu]$

$$
I=\int_{0}^{\nu} f(\omega) d \omega \ll I_{\nu}
$$

is much less than one of $I_{\nu}$, and one can neglect by it. Thus, for the integral between limits $[0, \infty]$, we have:

$$
\begin{aligned}
& \int_{0}^{\infty}\left\{\exp \left[-\frac{\left(\omega-\omega_{0}\right)^{2}}{4 \Gamma^{2}}\right]+\exp \left[-\frac{\left(\omega+\omega_{0}\right)^{2}}{4 \Gamma^{2}}\right]\right\}^{2} d \omega \\
= & \sqrt{2 \pi} \Gamma\left(1+\exp \left[-\frac{\omega_{0}^{2}}{2 \Gamma^{2}}\right]\right),
\end{aligned}
$$

and as a result

$$
\begin{aligned}
w_{m \perp} & =\frac{1}{40} \frac{V}{\sqrt{2 \pi}} \frac{\nu}{\Gamma}\left(\frac{\omega_{p} R_{\perp}}{c}\right)^{2}\left(\frac{R_{||}^{2}}{R_{||}^{2}+R_{\perp}^{2}}\right) \\
& \times\left(1+e^{-\frac{\omega_{0}^{2}}{2 \Gamma^{2}}}\right)\left|\mathbf{E}_{0}\right|^{2},
\end{aligned}
$$

provided that $\omega \gg \nu$.

(ii) Now we pass to the case of EM wave-polarization, where the magnetic field is directed along the main spheroid axis. In this case the scalar product in a second item under the integral in Eq. (41) is $\mathbf{H}(0, \omega) \mathbf{n}=$
$|\mathbf{H}(0, \omega)|$ and substituting Eq. (4) into Eq. (41), we come to the next expression

$w_{m \|}=\frac{V}{80} \frac{\pi}{\Gamma^{2}}\left(\frac{R_{\perp}}{c}\right)^{2}\left|\mathbf{E}_{0}\right|^{2} \int_{-\infty}^{\infty}\left[\sigma(\omega)+\sigma^{*}(\omega)\right] f(\omega) \omega^{2} \frac{d \omega}{2 \pi}$.

Using both the Eq. (42) and the result of the integration given by Eq. (47), we obtain finally the next expression for the energy absorbed with this polarization

$$
w_{m \|}=\frac{1}{80} \frac{V}{\sqrt{2 \pi}} \frac{\nu}{\Gamma}\left(\frac{\omega_{p} R_{\perp}}{c}\right)^{2}\left(1+e^{-\frac{\omega_{0}^{2}}{2 \Gamma^{2}}}\right)\left|\mathbf{E}_{0}\right|^{2},
$$

provided that $\omega \gg \nu$. The square of an amplitude of the electrical field in the previous expressions and further formally could be changed by $\left|\mathbf{E}_{0}\right|^{2} \rightarrow\left|\mathbf{H}_{0}\right|^{2}$, because the maximal value of the magnetic field in the pulse would be specified like similar one in Eq. (1), with taking into account that the relation between fields is given by Eq. (2).

If one suppose that a nanoparticle is irradiated by a plane EM wave, i.e., by the wave (1) with $\Gamma \rightarrow 0$, then the expressions (48) and (50) coincide asymptotically with the known results from our earlier works ${ }^{15.16}$, with an accuracy up to the constant $2 \sqrt{\pi / 2}$ [due to the distinction in a pulse forms (see previous note ${ }^{24}$ )].

\section{B. Kinetic approach}

When the nanoparticle size is less than the electron free pass in it, the phenomenological approach cannot be used anymore and ought to be changed by the kinetic approach. Note, that the latter permits to obtain the correct results for the case when the particle size is larger than the electron free pass as well. In the kinetic approach, the current $\mathbf{j}_{e d}(\omega)$ enters into Eq. (32), should be calculated by means of the formula (13). Using the nonequilibrium distribution function (33), one finds

$$
\begin{aligned}
w_{m} & =\frac{4}{m} \frac{e^{2}}{R^{4}}\left(\frac{m}{2 \pi \hbar}\right)^{3} R e \sum_{i j k l}^{3} R_{i} R_{j} R_{k} R_{l} \\
& \times \int_{-\infty}^{\infty} \frac{\alpha_{k j}^{*}(\omega) \alpha_{i l}(\omega)}{\nu-i \omega} \frac{d \omega}{2 \pi} \int_{-\infty}^{\infty} v_{j}^{\prime} v_{l}^{\prime} \delta\left(v^{2}-v_{F}^{2}\right) d^{3} v \\
& \times \int_{V^{\prime}} x_{k}^{\prime} x_{i}^{\prime}\left(1-e^{-(\nu-i \omega) t^{\prime}\left(r^{\prime}, v^{\prime}\right)}\right) d r^{\prime}
\end{aligned}
$$

where $v_{F}$ is the electron velocity on the Fermi sphere. We have omitted the item in Eq. (51) associated with the second term (with $v_{i}^{\prime}$ ) under the sum sign in Eq. (33). It is not difficult to show that the contribution of this term with integration over all electron coordinates will be equal to zero, because it is even with respect to coordinates, whereas an eddy field is an odd coordinate function. Then, the integration over all coordinates in 
Eq. (51), associated with the first term under the sum sign in Eq. (33), can be done exactly. We would write here only the final result. The details of calculations can be found in Ref. 15 .

$$
\begin{aligned}
& \int_{V^{\prime}} x_{k}^{\prime} x_{i}^{\prime}\left(1-e^{-(\nu-i \omega) t^{\prime}\left(r^{\prime}, v^{\prime}\right)}\right) d r^{\prime} \\
= & \pi R^{5}\left[2 \psi_{3} \frac{v_{k}^{\prime} v_{i}^{\prime}}{v^{\prime 2}}+\frac{\psi_{1}}{2}\left(\delta_{k i}-3 \frac{v_{k}^{\prime} v_{i}^{\prime}}{v^{\prime 2}}\right)\right] .
\end{aligned}
$$

Here,

$$
\begin{gathered}
\psi_{1}\left(v^{\prime}, \omega\right)=\frac{8}{15}-\frac{1}{q}+\frac{4}{q^{3}}-\frac{24}{q^{5}}+e^{-q} \frac{8}{q^{3}}\left(1+\frac{3}{q}+\frac{3}{q^{2}}\right), \\
\psi_{3}\left(v^{\prime}, \omega\right)=\frac{2}{5}-\frac{1}{q}+\frac{8}{3 q^{2}}-\frac{6}{q^{3}}+\frac{32}{q^{5}} \\
-e^{-q} \frac{2}{q^{2}}\left(1+\frac{5}{q}+\frac{16}{q^{2}}+\frac{16}{q^{3}}\right), \\
q=(\nu-i \omega) \frac{2 R}{v^{\prime}} .
\end{gathered}
$$

The next integration over all velocities space fails to be carried out in an analytical form for a common case. It can be fulfilled only if the frequency interval $[-\infty, \infty]$ to split arbitrarily into the two parts

$$
\int_{-\infty}^{\infty} \Xi(\omega) d \omega=2 \int_{0}^{\nu_{S}} \Xi_{L F}(\omega) d \omega+2 \int_{\nu_{S}}^{\infty} \Xi_{H F}(\omega) d \omega
$$

one of which will exceed, and another one will be less than the frequency associated with electron oscillations between particle walls

$$
\nu_{s}=v_{F} /(2 R) .
$$

The factor of 2 in Eq. (54) appear due to the fact that the integrants $\Xi(\omega)$ should be the even functions of $\omega$. The case with $\omega<\nu_{S}$ will be named further as the lowfrequency (LF) case, and the case with $\omega>\nu_{S}$ - as the highfrequency (HF) one. Let us consider first the latter. As a basis for it, we will apply the approximations

$$
\begin{gathered}
\operatorname{Re}\left\{\frac{1}{\nu-i \omega} \psi_{1}\left(v^{\prime}, \omega\right)\right\} \approx \frac{v^{\prime}}{2 R \omega^{2}}, \\
\operatorname{Re}\left\{\frac{1}{\nu-i \omega} \psi_{2}\left(v^{\prime}, \omega\right)\right\} \approx \frac{v^{\prime}}{8 R \omega^{2}},
\end{gathered}
$$

where

$$
\psi_{2}\left(v^{\prime}, \omega\right)=\psi_{3}\left(v^{\prime}, \omega\right)-\frac{3}{4} \psi_{1}\left(v^{\prime}, \omega\right)
$$

Details of calculations are presented in Refs. 15 and 16 . Here, we write down only the final result which can be obtained after integration over all electron velocities with the account of Eqs. (40) and (56)

$$
\begin{aligned}
w_{m, H F} & =\frac{9}{32} \frac{V}{4 \pi}\left(\frac{\omega_{p}}{c}\right)^{2} v_{F} R_{\perp} \\
& \times \int_{\nu_{S}}^{\infty}\left\{\eta_{m}^{H}\left(e_{s}\right)\left(\frac{R_{\|}^{2}}{R_{\| \mid}^{2}+R_{\perp}^{2}}\right)^{2}|\mathbf{H}(0, \omega)|^{2}+\left[\rho_{H}\left(e_{s}\right)-\eta_{m}^{H}\left(e_{s}\right)\left(\frac{R_{\| \mid}^{2}}{R_{||}^{2}+R_{\perp}^{2}}\right)^{2}\right]|\mathbf{H}(0, \omega) \mathbf{n}|^{2}\right\} \frac{d \omega}{2 \pi} .
\end{aligned}
$$

In expression (57), $\rho_{H}\left(e_{s}\right)$ and $\eta_{m}^{H}\left(e_{s}\right)$ are the functions depended only on the spheroid eccentricity $e_{s}$. Their analytical forms are given in Ref. 15. From Eq. (57), one can see that the frequency dependence of the integrand (for any given polarization) in the HF-case is contained only in the magnetic field amplitude. Practically this means that we can use the result of the integration obtained above in the phenomenological approach. If the lower integration limit in the phenomenological case was the value of $\nu$, then $\nu_{S}$ will play here the same role. It is not difficult to ensure that in the case with $\nu_{S}$, one can diminish the lower limit of integration to the zero as well. Hence, the energy of a magnetic component of a laser EM wave in (i) polarization (when the vector of the magnetic component is directed transverse to the long spheroid axis of revolution), with an account of the expressions (4) and (47) in Eq. (57), finally becomes

$$
\begin{aligned}
w_{m \perp H F} & =\frac{9}{32} \frac{V}{\sqrt{2 \pi}} \frac{\omega_{p}^{2}}{16 c^{2}} \frac{v_{F}}{\Gamma}\left(1+e^{-\frac{\omega_{0}^{2}}{2 \Gamma^{2}}}\right) \\
& \times \eta_{m}^{H}\left(e_{s}\right) R_{\perp}\left(\frac{R_{||}^{2}}{R_{||}^{2}+R_{\perp}^{2}}\right)^{2}\left|\mathbf{E}_{\mathbf{0}}\right|^{2},
\end{aligned}
$$

and for the (ii) polarization, when, vice verse, the vector 
of magnetic field component is directed along this axis, is represented by

$$
w_{m \| H F}=\frac{9}{32} \frac{V}{\sqrt{2 \pi}} \frac{\omega_{p}^{2}}{16 c^{2}} \frac{v_{F}}{\Gamma}\left(1+e^{-\frac{\omega_{0}^{2}}{2 \Gamma^{2}}}\right) \rho_{H}\left(e_{s}\right) R_{\perp}\left|\mathbf{E}_{\mathbf{0}}\right|^{2}
$$

Eqs. (58) and (59) coincide each with other for a spherical particle, because $\eta_{m}^{H}(0)=4 \rho_{H}(0)$. Besides, if one takes into consideration that the power of the absorbed energy is $W_{m}=w_{m} \Gamma$, where $w_{m}$ is given by Eqs. (58) or (59), and the parameter $\Gamma$ tends to the zero in $W_{m}$, then these equations will transform (with an accuracy of the constant of $2 \sqrt{\pi / 2}$ ) into the known ones from our previous calculations ${ }^{15,16}$ for a plane wave.

It remains to consider the LF-case. The calculation of Eq. (51) in this case can be done with the use of the next approximation

$$
\begin{aligned}
& \operatorname{Re}\left\{\frac{1}{\nu-i \omega} \psi_{1}\left(v^{\prime}, \omega\right)\right\} \approx \frac{R}{3 v^{\prime}} \\
& \operatorname{Re}\left\{\frac{1}{\nu-i \omega} \psi_{2}\left(v^{\prime}, \omega\right)\right\} \approx \frac{R}{36 v^{\prime}} .
\end{aligned}
$$

Furthermore, not complicated but cumbersome calculations lead us to the next result

$w_{m L F}=\frac{3}{16} V \frac{\omega_{p}^{2}}{4 \pi c^{2}} \frac{R_{\perp}^{3}}{v_{F}} \int_{0}^{\nu_{S}}\left\{\eta_{m}^{L}\left(e_{s}\right)\left(\frac{R_{||}^{2}}{R_{||}^{2}+R_{\perp}^{2}}\right)^{2}|\mathbf{H}(0, \omega)|^{2}+\left[\rho_{L}\left(e_{s}\right)-\eta_{m}^{L}\left(e_{s}\right)\left(\frac{R_{||}^{2}}{R_{||}^{2}+R_{\perp}^{2}}\right)^{2}\right]|\mathbf{H}(0, \omega) \mathbf{n}|^{2}\right\} \frac{d \omega}{2 \pi}$,

where $\rho_{L}\left(e_{s}\right)$ and $\eta_{m}^{L}\left(e_{s}\right)$ are some smooth functions of the spheroid eccentricity $e_{s}$. The behavior of these functions depending on the shape of the MN, which is specified by the ratio of $R_{\perp} / R_{||}$, one can find in Ref 15 . For a spherical particle

$$
\rho_{L}(0)=\rho_{H}(0)=2 / 3, \quad \eta_{m}^{L}(0)=\eta_{m}^{H}(0)=8 / 3 .
$$

For an estimation of Eq. (61), it is necessary to calculate the integral

$$
I_{L}=\int_{0}^{\nu_{s}} \omega^{2} f(\omega) d \omega
$$

It can be done analytically. It is easy to ensure, however, that similar to the calculation of the integral $I$ in Eq. (46), one obtains the same result using the integrant from Eq. (63) for $I_{L}$. In other words, one can get that

$$
I_{L} \ll I_{\nu_{S}}
$$

This means that in the sum of Eq. (54), one can neglect by the integral of $I_{L}$. In this case, for an arbitrary angle $\theta$ between direction of the magnetic field and the spheroid axis of revolution, we will obtain finally the expression

$$
w_{m S}=w_{m L F}+w_{m H F}=\frac{9}{64} \frac{V}{\sqrt{2 \pi}} \frac{\omega_{p}^{2}}{8 c^{2}} \frac{v_{F}}{\Gamma}\left(1+e^{-\frac{\omega_{0}^{2}}{2 \Gamma^{2}}}\right) R_{\perp}\left[\rho_{H}\left(e_{s}\right) \sin ^{2} \theta+\eta_{m}^{H}\left(e_{s}\right)\left(\frac{R_{||}^{2}}{R_{||}^{2}+R_{\perp}^{2}}\right)^{2} \cos ^{2} \theta\right]\left|\mathbf{E}_{\mathbf{0}}\right|^{2}
$$

The results (48) and (50) obtained in the phenomenological approach for a spherical nanoparticle go over into the ones described by Eqs. (58) and (59), or Eq. (65), obtained in the kinetic approach, if one performs formally the next replacement:

$$
\begin{aligned}
& \nu \rightarrow \frac{45}{64} \frac{v_{F}}{R_{\perp}}\left(\frac{R_{||}^{2}}{R_{||}^{2}+R_{\perp}^{2}}\right) \eta_{m}^{H}, \quad \text { for } \perp \text { polarization } \\
& \nu \rightarrow \frac{45}{32} \frac{v_{F}}{R_{\perp}} \rho_{H}, \quad \text { for } \| \text { polarization }
\end{aligned}
$$


of a spherical particle, the above substitution, obviously, takes the form:

$$
\nu \rightarrow \frac{15}{16} \frac{v_{F}}{R}, \quad \text { provided that } \omega \gg \nu
$$

\section{Results and Discussion}

Let us illustrate graphically the expressions obtained above analytically. We will calculate the ratio

$$
S_{m}=\frac{w_{m}}{2 w}
$$

between the energy absorbed by a unit volume of the $\mathrm{MN}$ and the energy traversed the nanoparticle, given by Eq. (23).

Studying the dependence of optical properties of nanoparticles on their shape, it would be worthwhile to compare the absorption for particles of various shapes but with equal volumes. The transformation of a particle shape can be described by the ratio of radiuses $R_{\perp} / R_{||}$. The condition of fixed particle volume $\left(V=\frac{4 \pi}{3} R_{\perp}^{2} R_{\|}=\right.$ const) with given ratio of $R_{\perp} / R_{||}$, defines the quantity of $R_{\perp}$ or of $R_{||}$. For instance,

$$
R_{\perp}=R\left(\frac{R_{\perp}}{R_{\|}}\right)^{1 / 3}, \quad R_{\|}=R\left(\frac{R_{\perp}}{R_{\|}}\right)^{-2 / 3}
$$

where $R$ is the radius of a sphere of an equivalent volume.

Let us study at first the dependence of $S_{m}$ on the particle shape. In Figs. 7 and 8 for various polarizations of EM wave, the dependence of $S_{m}$ on the degree of oblateness or prolateness of a spheroidal particle is depicted (at the frequency of the surface plasmon $\omega_{0}=\omega_{p} / \sqrt{3} \equiv \Omega$, which was a resonant one for a spherical particle in the case of the electric absorption). The calculations for $\perp$ and $\|$ polarizations were carried out using the next formulae

$$
\begin{gathered}
S_{m \perp}=\frac{\nu}{10 \pi} \Omega^{2}\left(\frac{R}{c}\right)^{3} \frac{\left(R_{\perp} / R_{\|}\right)^{2 / 3}}{1+\left(R_{\perp} / R_{\|}\right)^{2}}, \\
S_{m \|}=\frac{\nu \Omega^{2}}{20 \pi}\left(\frac{R}{c}\right)^{3}\left(R_{\perp} / R_{\|}\right)^{2 / 3},
\end{gathered}
$$

for phenomenological case, and

$$
\begin{gathered}
S_{m \perp}=\frac{9}{128 \pi} v_{F} \Omega^{2} \frac{R^{2}}{c^{3}} \eta_{m}^{H}\left(e_{s}\right) \frac{\left(R_{\perp} / R_{\|}\right)^{1 / 3}}{\left[1+\left(R_{\perp} / R_{\|}\right)^{2}\right]^{2}}, \\
S_{m \|}=\frac{9}{128 \pi} v_{F} \Omega^{2} \frac{R^{2}}{c^{3}} \rho_{H}\left(e_{s}\right)\left(R_{\perp} / R_{\|}\right)^{1 / 3},
\end{gathered}
$$

for kinetic one.
The calculation are performed assuming the next numerical parameters for $\mathrm{Au}$ particle: the electron concentration $^{23}$ of $5.9 \times 10^{22} \mathrm{~cm}^{-3}, \Omega=7.91 \times 10^{15} \mathrm{~s}^{-1}$, $\nu=3.39 \times 10^{13} \mathrm{~s}^{-1}$, and $v_{F}=1.39 \times 10^{8} \mathrm{sm} / \mathrm{s}$.

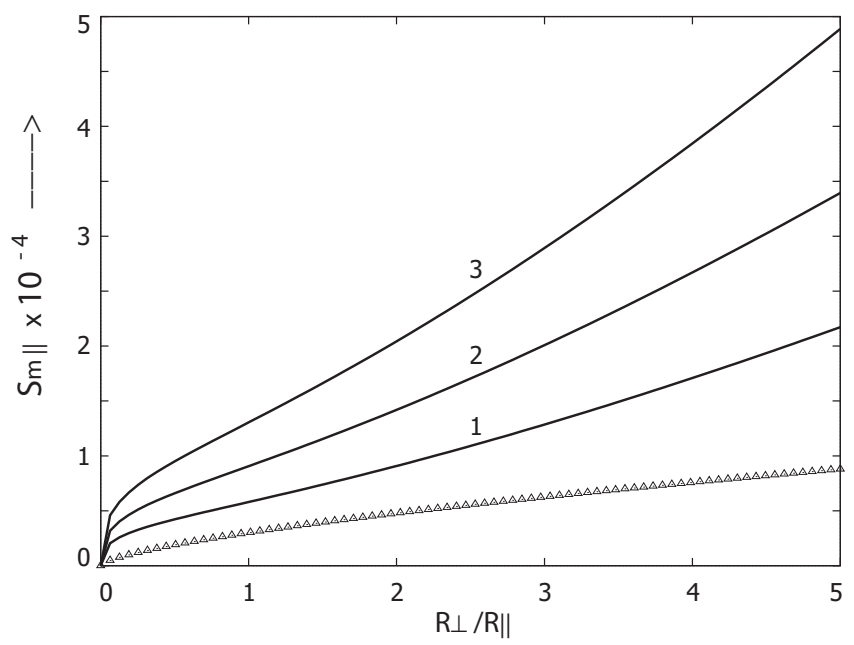

FIG. 7. The energy absorbed by Au particle for polarization of a magnetic field transverse $(\|)$ to it axis of revolution vs degree of spheroid oblateness or prolateness. Curves are obtained at the frequency of the plasmon resonance $\omega_{0}=\Omega$, for different $R, \AA$ : 200 (curve 1), 250 (2), 300 (3). The phenomenological dependence for $R=200$ Åis given by triangles.

The curves $1-3$ correspond to the different particle radiuses, whereas the curve of phenomenological dependencies builded on the base of the formulae (70) and (71) is given by triangles for $R=200 \AA$. Comparing curves $1-3$, it is clearly seen that the energy of laser pulses will be absorbed more strongly in Au particles of a larger volume. When the magnetic field polarization is directed along the MN rotation axis (I| polarization), the absorption grows with enhancing of the particle oblateness (Fig. 7), while for the transverse polarization of the magnetic field ( $\perp$ polarization), the absorption reaches the maximum for some values of the ratio of $R_{\perp} / R_{||}$(Fig. 8). In the phenomenological case, this maximum is reached for particles of a prolate shape when $R_{\perp} / R_{\|}=1 / \sqrt{2}$, and in the kinetic one - when $R_{\perp} / R_{\mid}=1 / \sqrt{11}$. If one compare the magnitude of the absorption maximum for the same pulse duration at $\perp$ polarization, which has been obtained in the phenomenological and the kinetic approaches from formulae (48) and (58), then one can get the relation

$$
\left.\frac{w_{m \perp, H F}}{w_{m \perp}}\right|_{\max } \simeq \frac{4}{5}\left(\frac{v_{F}}{\nu R}\right)
$$




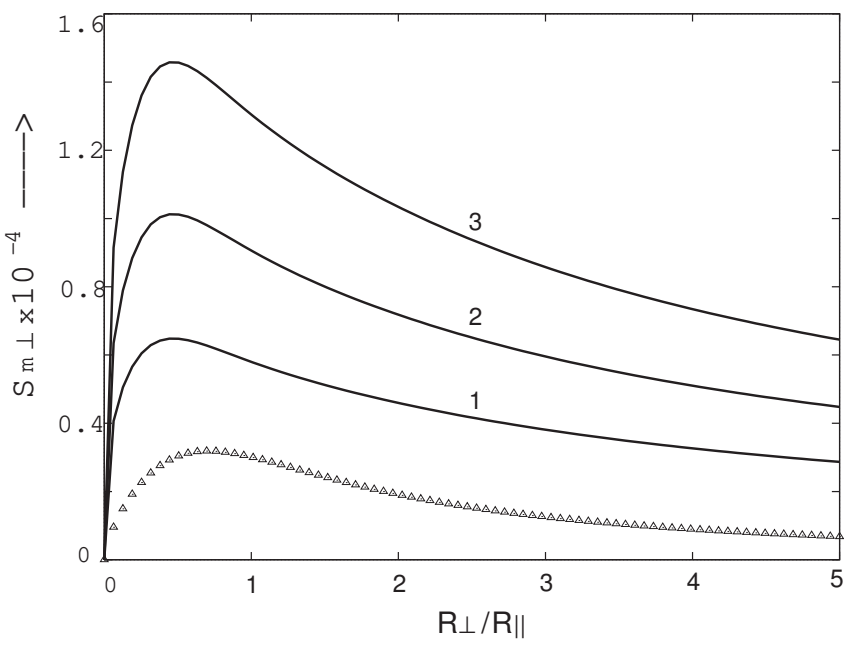

FIG. 8. The same, as in Fig. 7 for $\perp$ polarization of magnetic field.

From Eq. (74) it follows that this relation increases with the reduction of a particle radius and does not depend on laser beam parameters. The same one can see from Eqs. (70) - (73), where the ratio between absorbed and traversed energy in the case of magnetic absorption does not depend on parameter $\Gamma$. For $\mathrm{Au}$ particle with the radius, for instance, $200 \AA$ one can see (comparing curves 1 and those labeled by triangles in Fig. 8) that the absorption in the kinetic case is approximately of two times of magnitude higher.

With the extension of a MN prolateness, the magnitude of the absorption is decreased in the case of $\perp$ polarization of the magnetic field, and increased - in the case of $\|$ polarization. The reason for this is that the number of electron closed orbits is increased with growing of a prolateness of a particle in $\|$ polarization, and decreased in $\perp$ polarization of the magnetic field.

Contrary to the electric absorption, in the dependence of the absorbed energy on $R_{\perp} / R_{||}$, there does not occur neither the shift of maximum (for $\perp$ polarization) nor an appearance of any others maxima with the deviation of a carrier frequency from the frequency of the surface plasmon $\Omega$.

For the case when $\omega_{0}<\Omega$, the intensity of magnetic absorption slightly increases with decrease of $\omega_{0}$ and asymptotically approaches the constant value, when $\omega_{0}>\Omega$ [see Eqs. (58), (59), (48), and (50)]. This is true for both polarizations as well as for both the phenomenological and the kinetic approaches. But depending on the duration of an incident pulse, a certain peculiarities appears. Namely, the absorption intensity is increased with an enhancement of the pulse duration at different carrier frequencies for both examined here polarizations. This grows is more rapid at frequencies $\omega_{0} \ll \Omega$, but has a restriction connected with the evident fact that the space size of a pulse $c / \Gamma$ cannot exceed the length of the carrier wave $\lambda_{0}$ in vacuum: $\Gamma / \omega_{0} \geq 1 /(2 \pi) \approx 0.16$.

In contrast to spherical particles, the magnetic absorption in nonspherical ones drastically depends on magnetic field polarization. So, the absorption of a spheroidal MN increases for moderate prolate and decreases for oblate particles (comparing to the particles of a spherical shape of the same volume) at $\perp$ field polarization. It is the least for particles of an oblate shape. In the case of $\|$ polarization, vice versa: the absorption noticeably increases for an oblate and decreases for a prolate MN comparing to the spherical one. Similar trends holds true for phenomenological approach in both polarization as well.

If one choose a nanoparticle of a spheroidal shape with some fixed ratio of $R_{\perp} / R_{||}$and will change only the magnitude of the carrier frequency, then one can establish using Eqs. (58), (59), (48), and (50) for different polarizations of an incident field the following. (i) For MN of a prolate shape $\left(R_{\perp} / R_{||}<1\right)$ with an enhancement of a laser pulse duration at frequencies $\omega_{0} \ll \Omega$, the maximum of the energy absorption increases and does not split into the two peaks as we obtain above for prolate Au particle in an electric fields with $\Gamma$ exceeding some limit quantity (see Figs. 5 and 6). (ii) If the ratio of $\omega_{0} / \Omega$ is small, the rise of magnetic absorption reaches the higher point for the least frequency $\omega_{0}=\nu_{S}$. (iii) Comparing intensity of absorption for pulses of equal duration, but different polarization, one can ensure that more rapid enhancement of the energy absorption by MN with increasing of carrier frequency occurs at the $\perp$ polarization of the magnetic field.

For oblate particles, vice verse, an enhancement of the energy absorption by MN with decreasing of a carrier wave proceeds faster for the $\|$-polarization of the magnetic field, then for it $\perp$ polarization. The pulses of larger duration (with small value of $\Gamma$ ) are intensively absorbed at lower frequencies of a carrier wave in both polarizations.

Thus, the spheroidal MN of an oblate or prolate shape comparing to the spherical one can absorb the energy of the magnetic field from ultrashort laser pulses more or less intensively depending on the magnetic field orientation with respect to the spheroid axis of revolution.

It would be interesting also to monitor the absorption by a nanoparticle of a fixed shape with volume changing. This can be easy fulfilled for fixed ratio of $R_{\perp} / R_{||}$ with changing the radius of an equivalent sphere $R$ in the previously obtained formulae. Such one dependence for nonspherical particles can be strongly differ from an analogous one in the case of spherical particles and requires a special investigation. There is only problem, when the volume of particle is changed with simultaneous preservation of its shape, the size of a particle in same directions becomes greater than the electron free pass in those directions. Similar study for an electric absorption we have performed in Ref. 21.

From expressions (42) and (52), or (44) and (53) one can see only that the energy of the magnetic absorption with the use of the phenomenological description increases quadratically with $R_{\perp}$, whereas at the kinetic description, the growth proceeds linearly with the $R_{\perp}$. Generally, the increase of magnetic absorption with $R_{\perp}$ is due to the enhancement of the number of electron closed 
orbits with growing of a particle oblateness in both polarization of the magnetic field. The different behavior of the two approaches with $R_{\perp}$ can be understood from above Eq. (66), where phenomenological parameter $\nu$ is replaced by $v_{F} / R_{\perp}$ in a kinetic approach.

By making use of the above results, namely, Eq. (19) for the electric absorption and Eqs. (58) and (59) valid for the magnetic absorption in a high-frequency case, one can compare relative contributions for both polarizations.

$$
\begin{aligned}
\left(\frac{w_{m}}{w_{e}}\right)_{\|, \perp} & \simeq \frac{9}{16 \sqrt{2 \pi}} \frac{v_{F}}{c}\left(\frac{\Gamma R_{\perp}}{c}\right)\left(1+e^{-\frac{\omega_{0}^{2}}{2 \Gamma^{2}}}\right) e^{\frac{\left(\omega_{\|, \perp}-\omega_{0}\right)^{2}}{2 \Gamma^{2}}} \\
& \times\left\{\begin{array}{l}
\rho_{H}\left(e_{s}\right) \\
\eta_{m}^{H}\left(e_{s}\right)\left(\frac{R_{\mid}^{2}}{R_{||}^{2}+R_{\perp}^{2}}\right)^{2} .
\end{array}\right.
\end{aligned}
$$

For spherical particle, using Eq. (62), one can get that

$$
\left(\frac{w_{m}}{w_{e}}\right)_{s p h} \simeq \frac{3}{8 \sqrt{2 \pi}} \frac{v_{F}}{c}\left(\frac{\Gamma R}{c}\right)\left(1+e^{-\frac{\omega_{0}^{2}}{2 \Gamma^{2}}}\right) e^{\frac{\left(\omega_{p} / \sqrt{3}-\omega_{0}\right)^{2}}{2 \Gamma^{2}}}
$$

An estimation of the ratio of absorptions [from Eq. (76)] for particle with the radius of $R=50 \AA$, for which the input of the magnetic field overcomes the electric one for a separate absorption, ${ }^{21}$ shows that at the frequency $\omega_{0}=\Omega$ the absorption associated with an electric plasmon excitation is considerably higher than the magnetic one. The same one can see, comparing the magnitudes of absorption shown in Figs. 7 and 8 for magnetic absorption, with electric one (Fig. 2) for Au particle at plasmon resonances. However, the ratio between magnetic and electric fields input can enhanced with the pulse duration, and for plane monochromatic wave will be equal to 1 or even exceed 1, depending on the particle sizes. The substantial rice of this ratio at plasmon resonances can be realized only in $\|$ polarization of magnetic field for highly oblate particles. For instance, for Au particle of $R=200 \AA$, we can reach the same input of magnetic component as for electric one (with $\Gamma=2.637 \times 10^{15} \mathrm{~s}^{-1}$ ) at $R_{\perp} / R_{\|} \approx 800$. The input of a magnetic component in the absorption becomes comparable and even can exceed the electrical one, as well, if a carrier frequency $\omega_{0}$ deviates from the plasmon frequency $\Omega$, especially at small values of $\Gamma$. One can find from Eq. (176) that, e.g., for Au particle with $R=200 \AA$, and $\Gamma=1.13 \times 10^{15} \mathrm{~s}^{-1}$, at $\left|\Omega-\omega_{0}\right|=5 \times 10^{15} \mathrm{~s}^{-1}, w_{m} / w_{e} \simeq 1$.

In general, the absorption of energy from ultrashort laser pulses by spheroidal MN depends both on the particle and the pulse characteristics. If the parameters of laser pulses are given, the absorption by MN depends on its volume (for fixed shape) as well as on the its shape (for fixed volume). We have especially interested in the dependence on the particle shape, because with changing of it, we can variate the position of plasmon resonances on the frequency scale. In other words, with changing the ratio of $R_{\perp} / R_{\|}$, the plasmon resonances may came close to or move away from the fixed carrier frequency. That is important for the amount of energy absorbed by particle. Such an approach is very demonstrable and productive for a theoretical studding of the optical properties of a MN. But for direct comparison of a theoretical predictions with an experiments it is necessary to have the data for a metallic nanoparticles of equal volumes but different in their shapes.

\section{CONCLUSIONS}

We develop the theory of the absorption of ultrashort laser pulses of a different duration and carrier frequencies by small metallic particles of a spheroidal shape. The cases when an electron free pass is greater or much less than a particle size are considered. There has been analyzed the dependence of the absorbed energy on the degree of a particle oblateness or prolateness at the frequency of a plasmon resonance and at frequencies that are greater or less than it. The simple analytic expressions are obtained, which make it possible to study the plasmon and magnetic absorption of laser pulses depending on the duration of these pulses, the magnitude of the carrier frequency, the particle shape, and the field polarizations. The analysis of the dependence of the energy absorbed by a spheroidal MN on the degree of its prolateness or oblateness was carried out for different values of pulse duration and frequencies that are higher, lower, or equal to the plasmon resonance frequency.

At the frequency of a carrier wave, which is equal with that of a surface plasmon, the maximum absorption was observed for spherical MNs. As soon as the carrier frequency deviates from that of the surface plasmon in a spherical particle, the two maxima appear in the dependence of the absorbed energy on the ratio of spheroid semiaxes: one of them corresponds to the prolate particles, while the other - to oblate particles. As the frequency deviates from the resonance one, the peak of the absorbed energy at first decreases in an absolute value, then splits and finally stabilizes for particles being more and more prolate or oblate.

For the case of spheroidal MNs subjected to the laser irradiation, with increase in the pulse duration, the peak in the dependence of the absorption on the frequency of a carrier wave also splits into two peaks located on opposite sides of the resonance (for a spherical particle) frequency. The double peaks originate from the resonances excited by ultrashort laser pulses at the frequencies of plasmon oscillations along or transverse to the spheroid rotation axis. A distance from the minimum in a well between the doublet peaks to the frequency of a plasmon resonance for a spherical particle provides the information about the degree of prolateness or oblateness of a nanoparticle.

As the degree of prolateness or oblateness increases, the distance between the doublet components grows. At the fixed incident angle of a laser pulse, the peak height keeps constant, with its value being dependent only on 
the pulse duration. It was considered how the change in the incident angle of a laser pulse directed along or transverse to the spheroid rotation axis effects the value of the relative absorption intensity.

We have found that the absorption in nonspherical particles drastically depends on the magnetic field polarization with respect to the particle rotation axis. Namely, for $\mathrm{MN}$ of a spheroidal shape, with the direction of the magnetic field transverse to the spheroid axis of revolution ( $\perp$ polarization), the absorption is increased for a prolate and decreased for an oblate MN compared to a particle of a spherical shape of the same volume. The case of the magnetic field orientation along the axis of revolution of a particle (I| polarization) proved to be the contrary one: the absorption is increased for a oblate MN and decreased for a prolate one.

It appears that the energy of laser pulses with the larger value of $1 / \Gamma$ is absorbed by $\mathrm{MN}$ better than with the smaller one. In the case with increasing of a particle oblateness, the magnetic absorption is increased for longitudinal $(||)$, and decreased - for a transverse $(\perp)$ polarization of the magnetic field. Comparing to the MN of spherical shape, the magnetic absorption for nanoparticles of a prolate shape is increased with rising of particle prolateness at $\perp$ polarization of magnetic field, reaching maximum for the ratio of spheroid hal- faxes $R_{\perp} / R_{||} \approx 7 / 15$, and diminishes for the larger prolateness. At $\|$ polarization, increasing in a prolateness leads to decrease in the absorption.

In the kinetic approach, the maximum value of the magnetic absorption for a fixed pulse duration and at $\perp$ polarization of magnetic field can exceed by two times or more (for particles with $R \leq 200 \AA$ ) the absorption, obtained in the phenomenological approach [at $\left.T=0^{\circ} \mathrm{C}\right]$.

With reduction in a carrier frequency, the intensity of the magnetic absorption by $\mathrm{MN}$ of a prolate shape is increased for $\perp$ polarization of the magnetic field more distinctly than at it $\|$ polarization. As soon as it took place, the pulses with longer duration are absorbed better at the lowest frequencies of the carrier wave. For oblate particles, on the contrary, the faster enhancement of the energy absorption by MN with increasing carrier frequency occurs for $\|$ polarization of the magnetic field, than at it $\perp$ polarization.

For frequencies of carrier wave close to the plasmon resonances in spheroidal $\mathrm{MN}$, the electric absorption is considerably higher than the magnetic one at $\perp$ polarization of magnetic field and can be comparable with one at || polarization for highly oblate MN.

A comparison between the results obtained in both the phenomenological and kinetic approaches have been advanced.
1 D. Boyer, P. Tamarat, A. Maali et al., Science 297, 1160 (2002).

2 P. Alivisatos, Nature Biotechnology 22, 51 (2004); J. Zhao, L.J. Sherry, G.C. Schatz, and R.P. Van Duyne, Molecular Plasmonics 14, 1418 (2008).

${ }^{3}$ F. Stietz, J. Bosbach, T. Wenzel et al., Phys. Rev. Lett. 84, 5644 (2000); B. Lamprecht et al., Appl. Phys. B 69, 223 (1999).

4 A. Arbouet, D. Christofilos, N. Del Fatti et al., Phys. Rev. Lett. 93, 127401 (2004).

5 S. Berciaud et al., Nano Lett. 5, 515 (2005).

6 M. Perner, P. Bost, U. Lemmer et al., Phys. Rev. Lett. 78, 2192 (1997).

7 J.-Y. Bigot, V. Halte, J.-C. Merle, and A. Daunois, Chem. Phys. 251, 181 (2000); M. Bauer and M. Aeschlimann, J. Electron. Spectrosc. Relat. Phenom. 124, 225 (2002).

8 J.-C. Diels and W. Rudolph, Ultrashort Laser Pulse Phenomena (Academic, New York, 1996).

9 A.B. Shvartsburg, Time-Domain Optics of Ultrashort Waveforms (Oxford, Clarendon Press, 1996).

10 U. Kreibig and M. Vollmer, Optical Properties of Metal Clusters (Springer, Berlin, 1995).

11 W.A. de Heer, Rev. Mod. Phys. 65, 611 (1993).

12 E. Hao and G.C. Shatz, J. Chem. Phys. 120, 357 (2004).

13 J.W. Haus et al., J. Appl. Phys. 65, 1420 (1989).

14 G. Weick, R.A. Molina, D. Weinmann, and R.A. Jalabert, Phys. Rev. B 72, 115410 (2005).

15 P.M. Tomchuk and N.I. Grigorchuk, Phys. Rev. B 73, 155423 (2006).
16 P.M. Tomchuk and N.I. Grigorchuk, Ukr. Phyz. J., 52, 889 (2007); N.I. Grigorchuk and P.M. Tomchuk, Zh. Fiz. Doslid. 12, 2701 (2008).

17 L.D. Landau and E.M. Lifshits, Electrodynamics of Continuous Media (Pergamon, New York, 1984).

18 H. Bremermann, Distributions, Complex Variables, and Fourier Transforms (Addison-Wesley, Reading, 1965).

19 A. Kawabata and R. Kubo, J. Phys. Soc. Jpn. 21, 1765 (1966).

20 R.A. Molina, D. Weinmann, and R.A. Jalabert, Phys. Rev. B 65,155427 (2002).

21 N.I. Grigorchuk and P.M. Tomchuk, Fiz. Nizk. Temp. 33, 1119 (2007);33, 461 (2007).

22 G. Arfken, Mathematical Methods for Physicists (Acad. Press, New York, 1985).

23 C. Kittel, Introduction to Solid State Physics, 6th ed. (Wiley, New York, 1986).

24 This dependence appears in Eq. (16) from $\gamma$ which depends on $R$ due to the conductivity tensor (18)

25 W. Ekardt, Phys. Rev. B 32, 1961 (1985).

26 The multiplier $1 / 2$ appears as a result of different choices of the expressions for the EM wave field: in work 15, we set it as $\mathbf{E}_{0} \exp [i(\omega t-\mathbf{k r})]$; but, here at $\Gamma \rightarrow 0$, we have $\mathbf{E}_{0} \cos \left(\omega_{0} t-\mathbf{k}_{0} \mathbf{r}\right)$ according to formula (1). The another multiplier $\sqrt{\pi / 2}$ results from the fact that we deal here with the Gaussian-like, rather than rectangular pulse.

27 G.W Kaye, T.H. Laby, Tables Physical and Chemical Constants (Longmans, London, New York, Toronto, 1961). 